\title{
An Experimental Evaluation of Mixup Regression Forests
}

Rodriguez, Juan; Juez-Gil, Mario; Arnaiz-Gonzalez, Alvar; Kuncheva, Ludmila

\section{Expert Systems with Applications}

\author{
DOI: \\ https://doi.org/10.1016/j.eswa.2020.113376
}

Published: 01/08/2020

Peer reviewed version

Cyswllt i'r cyhoeddiad / Link to publication

Dyfyniad o'r fersiwn a gyhoeddwyd / Citation for published version (APA):

Rodriguez, J., Juez-Gil, M., Arnaiz-Gonzalez, A., \& Kuncheva, L. (2020). An Experimental Evaluation of Mixup Regression Forests. Expert Systems with Applications, 151, [113376]. https://doi.org/10.1016/j.eswa.2020.113376

\footnotetext{
Hawliau Cyffredinol / General rights

Copyright and moral rights for the publications made accessible in the public portal are retained by the authors and/or other copyright owners and it is a condition of accessing publications that users recognise and abide by the legal requirements associated with these rights.

- Users may download and print one copy of any publication from the public portal for the purpose of private study or research.

- You may not further distribute the material or use it for any profit-making activity or commercial gain

- You may freely distribute the URL identifying the publication in the public portal ?
}

Take down policy

If you believe that this document breaches copyright please contact us providing details, and we will remove access to the work immediately and investigate your claim. 


\title{
An Experimental Evaluation of Mixup Regression Forests
}

\author{
Juan J. Rodríguez ${ }^{\mathrm{a}}$, Mario Juez-Gila ${ }^{\mathrm{a}}$ Álvar Arnaiz-González ${ }^{\mathrm{a}}$, Ludmila I. Kuncheva ${ }^{\mathrm{b}}$ \\ ${ }^{a}$ Escuela Politécnica Superior, Universidad de Burgos, 09006 Burgos, SPAIN \\ ${ }^{b}$ School of Computer Science and Electronic Engineering, Bangor University, Dean Street, Bangor LL57 \\ $1 U T, U K$
}

\begin{abstract}
Over the past few decades, the remarkable prediction capabilities of ensemble methods have been used within a wide range of applications. Maximization of base-model ensemble accuracy and diversity are the keys to the heightened performance of these methods. One way to achieve diversity for training the base models is to generate artificial/synthetic instances for their incorporation with the original instances. Recently, the mixup method was proposed for improving the classification power of deep neural networks (Zhang et al., 2017). Mixup method generates artificial instances by combining pairs of instances and their labels, these new instances are used for training the neural networks promoting its regularization. In this paper, new regression tree ensembles trained with mixup, which we will refer to as Mixup Regression Forest, are presented and tested. The experimental study with 61 datasets showed that the mixup approach improved the results of both Random Forest and Rotation Forest.
\end{abstract}

Keywords: Mixup, Regression, Random Forest, Rotation Forest

\section{Introduction}

The idea that motivates this study, in relation to problems that ensemble techniques 3 can solve, is that an increase in base-model diversity will improve ensemble performance, 4 generalization, and robustness. Diversity is a key attribute of an ensemble, without which 5 ensemble methods would not be as successful as they are (Kuncheva \& Whitaker, 2003). It

Email addresses: jjrodriguez@ubu.es (Juan J. Rodríguez), mariojg@ubu.es (Mario Juez-Gil), alvarag@ubu.es (Álvar Arnaiz-González), 1.i.kuncheva@bangor.ac.uk (Ludmila I. Kuncheva) 
can be achieved in several ways: by using different methods for building the classifiers in the ensemble (heterogeneous ensemble), by using methods that build classifiers with random components, and by using different training sets. The focus of this paper rests on the last strategy, in particular, in making new instances that not found in the original set for creating different training sets.

Mixup has recently been proposed by Zhang et al. (2017) for training deep neural networks using combinations of pairs of examples and their labels. Given a training set where each example is $(x, y)$, with an input, $x$, and a corresponding output, $y$, then the combined examples $(\tilde{x}, \tilde{y})$ are generated as

$$
\begin{aligned}
& \tilde{x}=\lambda x_{i}+(1-\lambda) x_{j} \\
& \tilde{y}=\lambda y_{i}+(1-\lambda) y_{j}
\end{aligned}
$$

where $\left(x_{i}, y_{i}\right)$ and $\left(x_{j}, y_{j}\right)$ are two examples, drawn at random from the training data, and $\lambda \in[0,1]$. The values of $\lambda$ were obtained using the Beta distribution: $\lambda \sim \operatorname{Beta}(\alpha, \alpha)$, with $\alpha \in(0, \infty)$.

Some example mixup data projections can be seen in figures 1 and 2. Figure 1 shows a single input dataset where the input variable and the output variable are represented on the $x$ axis and the $y$ axis, respectively, and the instances are generated with mixup. Figure 2 shows a couple of examples: two two-input datasets and the mixup-generated instances. The output values of the original datasets are in $\{-1,1\}$ and the output values of the datasets that are generated are in $[-1,1]$. Figure 3 shows the predictions of a single random tree for the datasets shown in Figure 2.

Mixup differs from other data augmentation approaches, in so far as its outputs are also combined. The combination of the outputs to address regression problems is a straightforward procedure.

As shown in Figure 1, some of the examples generated with mixup are clearly noise. Although it can be detrimental, noise injection has previously been used as a strategy for building successful ensembles (Melville \& Mooney, 2005; Frank \& Pfahringer, 2006; Martínez-Muñoz \& Suárez, 2005; Gónzalez et al., 2017). In mixup forests, the prevalence 

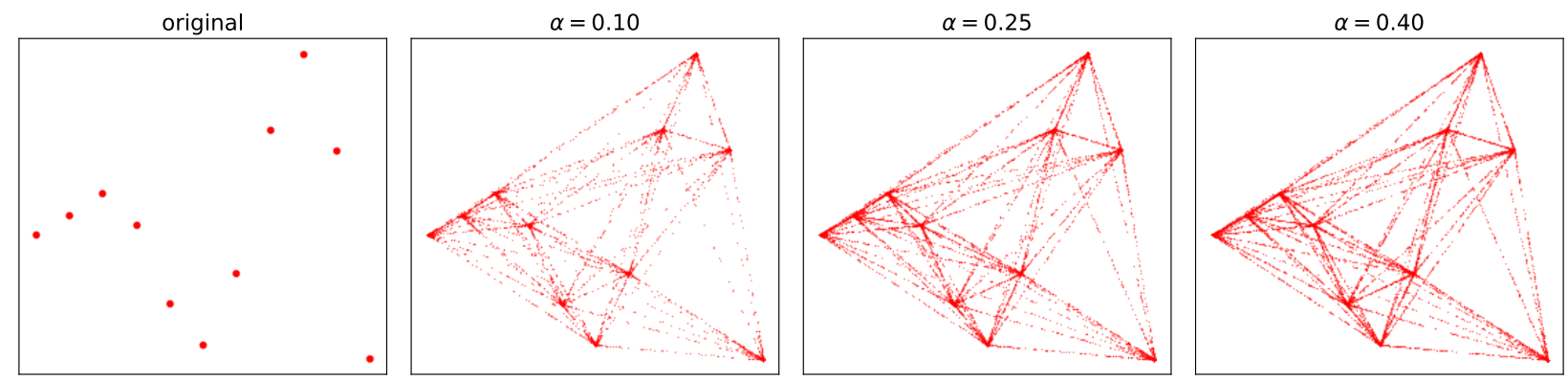

Figure 1: A regression problem dataset with a single input ( $x$ axis), and a single continuous output ( $y$ axis). Artificial instances are generated with mixup for $\alpha \in\{0.1,0.25,0.4\}$.
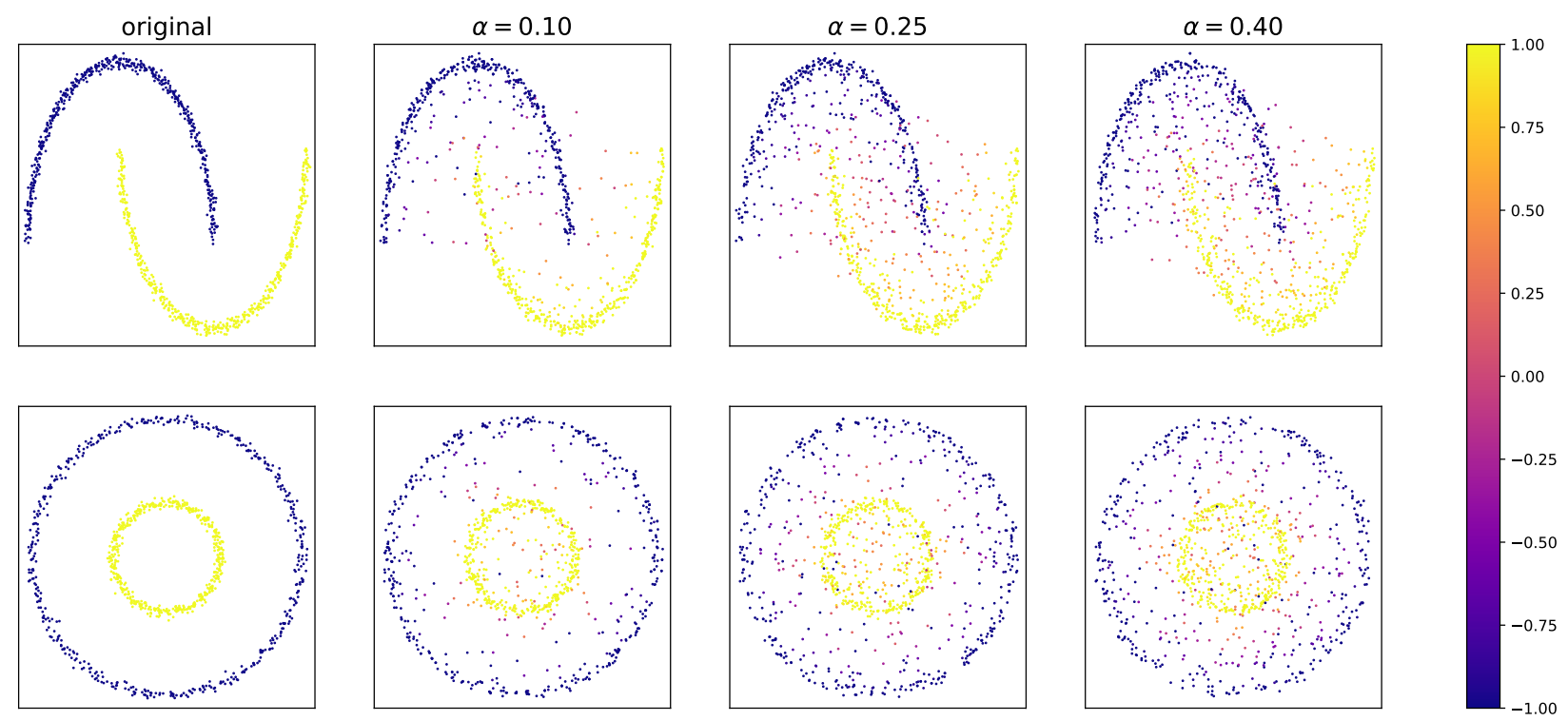

Figure 2: Two two-inputs datasets and the datasets generated with mixup for $\alpha \in\{0.1,0.25,0.4\}$. The output variables are shown in yellow and in blue. 

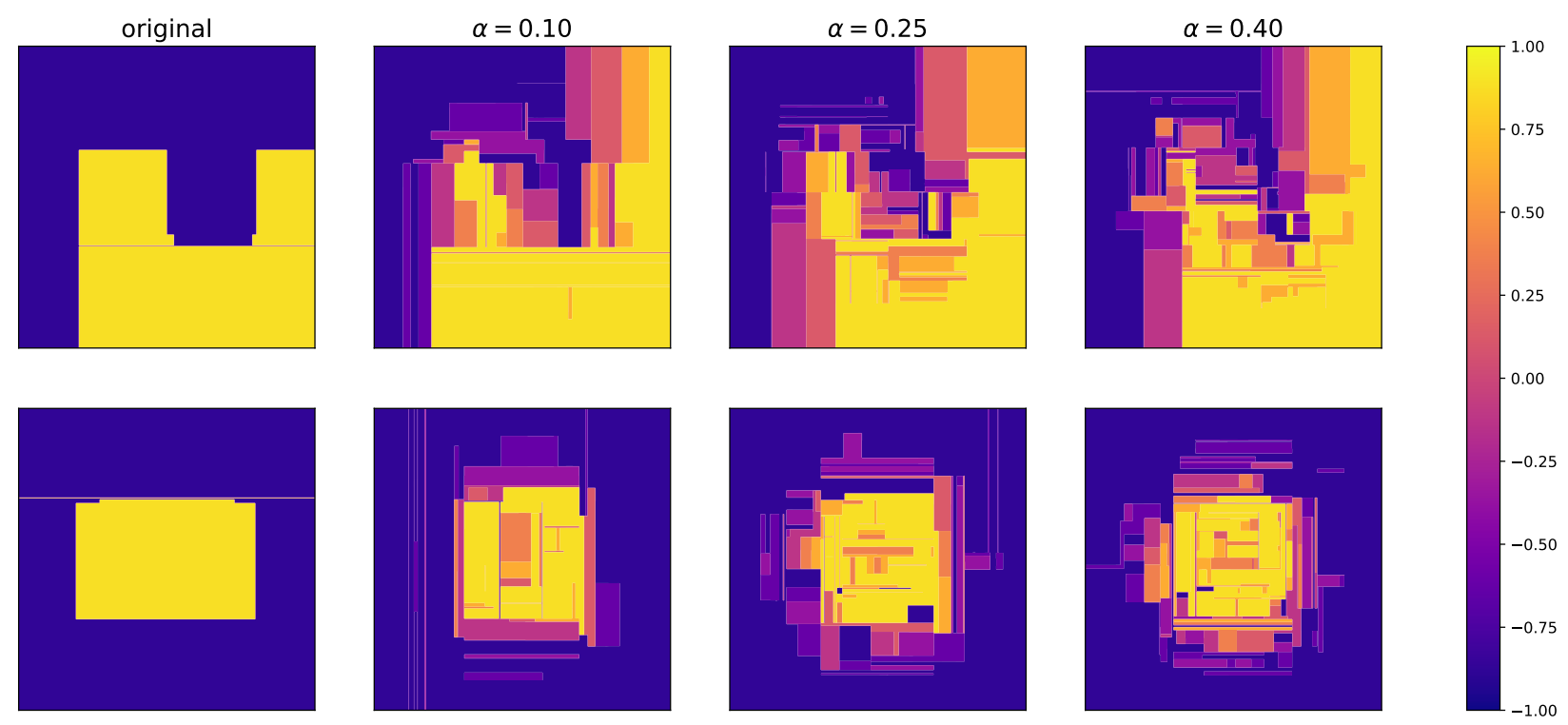

Figure 3: Predictions given by a single random tree trained with the corresponding datasets from Figure 2.

of these noisy examples can be controlled with the $\alpha$ value and the number of artificial examples that are generated.

Ensemble techniques have successfully been applied in various domains over the past few decades. Many works and several literature reviews have been published on both classification (Kuncheva, 2014) and regression (Mendes-Moreira et al., 2012) ensembles. Some illustrative examples of ensemble applications are detailed below.

In industrial environments, ensembles can be used as predictive models with adaptive capabilities, for example, to respond to incidences at processing plants (Soares \& Araújo, 2015). Financial forecasting with ensembles has also been a very frequent research topic, among other examples, for the prediction of trading in stocks (Weng et al., 2018) and bankruptcy trends (Chen et al., 2020). It is also of great industrial interest, for example, in the construction industry, where ensembles have been used for the prediction of financial distress (Choi et al., 2018). Many techniques for credit risk assessment have been proposed, based on both statistics and Artificial Intelligence (AI) models; a task in which ensembles have demonstrated good performance (Marqués et al., 2012). In biometrics, improved recognition rates can be achieved using multimodal biometric systems that capture multiple biometric traits, 
e.g. fingerprint, iris and facial features; multimodal data learning in those fields can be addressed by using ensembles (Ross \& Jain, 2003). The advantages and the convenience of ensemble learning to learn from multimodal features have likewise benefited several clinical practices (Tay et al., 2013). The sort of highly robust system required for image recognition tasks, such as facial recognition, can be provided by ensembles, to address the diversity of facial expressions and aging effects (Sirlantzis et al., 2008). Real-life problems, such as spam detection (Geng et al., 2007), translation of DNA sequences (García-Pedrajas et al., 2012), and the detection of credit-card fraud (Panigrahi et al., 2009), are known as imbalanced learning problems that can also be solved using ensemble techniques (Galar et al., 2012). The mixup data augmentation strategy proposed in this paper, might therefore lead to even better ensemble models for the aforementioned applications, as the artificial generation of instances has the potential to improve the performance of almost any ensemble method.

The contribution of this study relates to the novel use of the mixup approach. It demonstrates that artificial examples generated by mixup contribute to improved ensemble performance in regression tasks. Mixup is therefore considered for regression, mainly because of its simplicity: it can be used with all data types and needs no adjustments to the model.

The rest of the paper will be organized as follows. In section 2, a brief literature review of the most relevant works in this field will be presented. In section 3, the experimental setup will be described. Then the results will be presented and analyzed in Section 4. Finally, some concluding remarks and suggestions for future research work will be outlined in section 5 .

\section{Related works}

Diversity between the members of an ensemble means that those ensembles are capable of better predictions than the individual ensemble members. One way to achieve diversity is by introducing artificial examples for training, for example through the mixup approach. Data augmentation with artificial examples has previously been used in many ensemble algorithms, some of which are detailed below.

In DECORATE (Diverse Ensemble Creation by Oppositional Relabeling of Artificial Training Examples) (Melville \& Mooney, 2003, 2005), instances are generated based on the 
distribution of the data. The labels of the new instances are assigned with a probability that is proportional to the inverse of the probability assigned by the current ensemble, because the purpose of the artificial instances is to increase diversity. In Bagging with Input Smearing (Frank \& Pfahringer, 2006), the generation of artificial instances add noise to actual instances.

In imbalanced classification problems ${ }^{1}$, artificial examples are commonly used for increasing the number of instances of the minority class/es. As with mixup, in SMOTE (Chawla et al., 2002), artificial instances are also obtained by combining pairs of instances. In this case, as both instances in a pair are of the same class, the label of the artificial instances is the same as the instances used to generate them. SMOTE was not originally proposed as an ensemble method and can in fact be used as a pre-processing step before the construction of a model. Nevertheless, it can also be directly used in ensembles, by training each base classifier with a different set of original and artificial instances. SMOTE has been combined with generic ensemble methods giving rise to SMOTEBoost (Chawla et al., 2003) and SMOTEBagging (Wang \& Yao, 2009), among others.

There are many other methods for balancing datasets by augmenting the minority classes with artificial instances (Han et al., 2005; He et al., 2008; Menardi \& Torelli, 2014; Zhu et al., 2017). Some of these methods, such as SMOTE, have also been adapted to regression problems (Torgo et al., 2013).

Likewise, highly sophisticated approaches exist for augmenting datasets. Most of those have been specifically designed for a given data type, for example, images (Tokozume et al., 2017, 2018; Inoue, 2018; Summers \& Dinneen, 2019). Such approaches require training and adjusting a model, in order to generate the artificial instances (Mayo \& Frank, 2017; Verma et al., 2018; Guo et al., 2018; Lindenbaum et al., 2018; Beckham et al., 2019).

Here, mixup was chosen as the simplest augmentation method and the significant advantage of its use with regression ensembles of random trees (Mixup Regression Forests) will be demonstrated in the following section.

\footnotetext{
${ }^{1}$ Imbalanced classification problems are those related to datasets and domains where one class has a much greater number of examples than another (Haixiang et al., 2017).
} 


\footnotetext{
${ }^{2}$ http://www.cs.waikato.ac.nz/ml/weka/index_datasets.html

${ }^{3}$ http: //www.dcc.fc.up.pt/ ltorgo/Regression/DataSets.html
} 


\begin{tabular}{|c|c|c|c|c|c|c|c|}
\hline Dataset & Examples & Numeric & Nominal & Dataset & Examples & Numeric & Nominal \\
\hline 2d-planes & 40768 & 10 & 0 & house- $16 \mathrm{H}$ & 22784 & 16 & 0 \\
\hline abalone & 4177 & 7 & 1 & house-8L & 22784 & 8 & 0 \\
\hline ailerons & 13750 & 40 & 0 & housing & 506 & 12 & 1 \\
\hline auto-horse & 205 & 17 & 8 & hungarian & 294 & 6 & 7 \\
\hline auto-mpg & 398 & 4 & 3 & kin8nm & 8192 & 8 & 0 \\
\hline auto-price & 159 & 15 & 0 & longley & 16 & 6 & 0 \\
\hline auto93 & 93 & 16 & 6 & lowbwt & 189 & 2 & 7 \\
\hline bank-32nh & 8192 & 32 & 0 & machine-cpu & 209 & 6 & 0 \\
\hline bank-8FM & 8192 & 8 & 0 & mbagrade & 61 & 1 & 1 \\
\hline baskball & 96 & 4 & 0 & meta & 528 & 19 & 2 \\
\hline bodyfat & 252 & 14 & 0 & $\mathrm{mv}$ & 40768 & 7 & 3 \\
\hline bolts & 40 & 7 & 0 & $\mathrm{pbc}$ & 418 & 10 & 8 \\
\hline breast-tumor & 286 & 1 & 8 & pharynx & 195 & 1 & 10 \\
\hline cal-housing & 20640 & 8 & 0 & pole & 15000 & 48 & 0 \\
\hline cholesterol & 303 & 6 & 7 & pollution & 60 & 15 & 0 \\
\hline cleveland & 303 & 6 & 7 & puma32H & 8192 & 32 & 0 \\
\hline cloud & 108 & 4 & 2 & puma8NH & 8192 & 8 & 0 \\
\hline cpu & 209 & 6 & 1 & pw-linear & 200 & 10 & 0 \\
\hline cpu-act & 8192 & 21 & 0 & pyrimidines & 74 & 27 & 0 \\
\hline cpu-small & 8192 & 12 & 0 & quake & 2178 & 3 & 0 \\
\hline delta-ailerons & 7129 & 5 & 0 & schlvote & 38 & 4 & 1 \\
\hline delta-elevators & 9517 & 6 & 0 & sensory & 576 & 0 & 11 \\
\hline detroit & 13 & 13 & 0 & servo & 167 & 0 & 4 \\
\hline diabetes-numeric & 43 & 2 & 0 & sleep & 62 & 7 & 0 \\
\hline echo-months & 130 & 6 & 3 & stock & 950 & 9 & 0 \\
\hline elevators & 16599 & 18 & 0 & strike & 625 & 5 & 1 \\
\hline elusage & 55 & 1 & 1 & triazines & 186 & 60 & 0 \\
\hline fishcatch & 158 & 5 & 2 & veteran & 137 & 3 & 4 \\
\hline friedman & 40768 & 10 & 0 & vineyard & 52 & 3 & 0 \\
\hline fruitfly & 125 & 2 & 2 & wisconsin & 194 & 32 & 0 \\
\hline gascons & 27 & 4 & 0 & & & & \\
\hline
\end{tabular}

Table 1: Experimental dataset characteristics. 


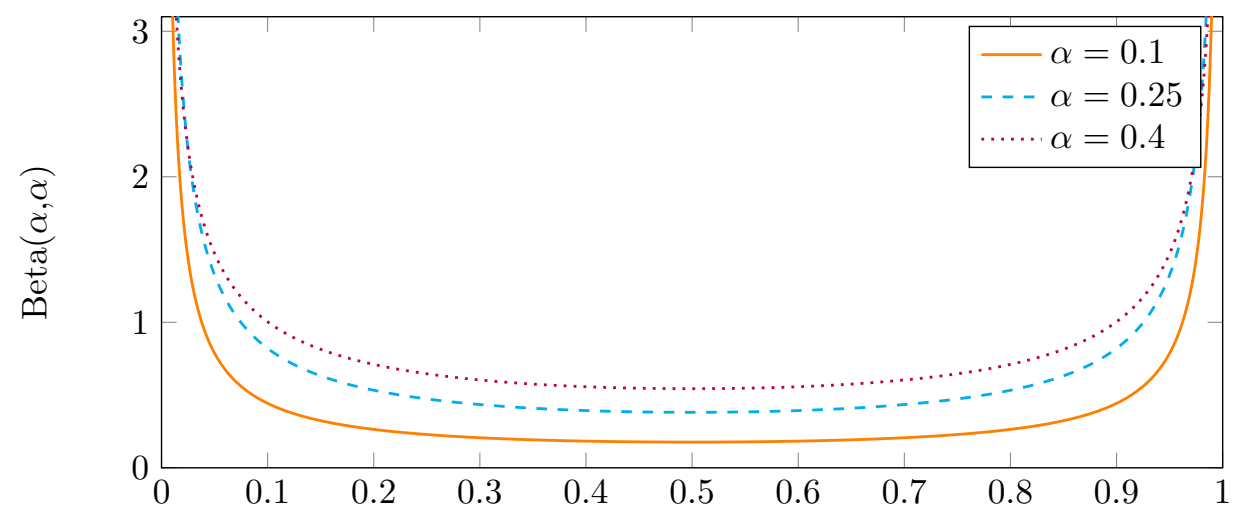

Figure 4: Beta distribution of the $\alpha$ values under consideration.

\subsection{Settings}

The experiments were performed using Weka (Hall et al., 2009). The default parameter's values of Random Forest and Rotation Forest were used, unless otherwise specified. For Random Forest, the default number of random attributes is $\log _{2}(m)+1$ where $m$ is the number of attributes. For Rotation Forest, the default size for each group of attributes is 3. The default method for constructing the trees in Rotation Forest, which only works for classification, is J48. Hence, REPTree, a tree method for regression, was used with no pruning, as ensembles generally work better with unstable models and pruning increases stability.

The results were generated using a $5 \times 2$-fold cross validation. The reported values are therefore averaged values from the 10 experiments. Three performance measures were calculated: RMSE (Root Mean Squared Error), MAE (Mean Absolute Error), and correlation.

The size of each ensemble was set at 100. The number of artificial examples to be generated was set at $50 \%$ of the training data size. Three values were applied $(0.10,0.25$, and 0.40 ) for the $\alpha$ values (in the Beta distribution), from the recommended range of $[0.1,0,4]$ in (Zhang et al., 2017). Figure 4 plots the Beta distribution for these $\alpha$ values.

One option for using mixup with nominal attributes is to transform them into numeric attributes. For example, one approach is to turn them into numerical values (that introduces an artificial order), and another is to turn them into binary attributes (greatly multiplying the attributes when there are many nominal values per attribute). Nevertheless, the mixing 
of two nominal value attributes was done in the experiments, by randomly selecting a single one. The probability of selecting the first nominal value is $\lambda$.

The number of artificial examples and the $\alpha$ value are hyper-parameters that can potentially improve the results when adjusted for each dataset.

\section{Results and discussion}

Tables 2, 3, and 4 show the results for RMSE, for MAE, and for correlation, respectively.

Pairwise comparisons. Tables 5 and 6 show the number of datasets for which the column method achieved better results than the row method. As 61 datasets were used in the experiments, a value greater than or equal to 31 will indicate that the column method has better results than the row method. It can be seen that the results are favorable for variants with mixup, especially for RMSE and correlation.

Relative scores. Figure 5 shows the boxplots of the relative scores, comparing the original method (Random or Rotation Forest) with the variants with mixup. The relative score for a given measure is defined as $(b-a) / a$ where $a$ and $b$ represent the performance of the original method and the performance of the variant method, respectively. When the measure is an error (RMSE or MAE), negative values of the score indicate that the variant is better. In contrast, positive values for correlation indicate that the variant is better. Each boxplot was obtained from the relative scores of the 61 datasets. The outliers were not included in the boxplots for the relative scores, as their inclusion would leave the boxes very small, because the relative scores of these few datasets (outliers) are much larger.

The boxplots and the signs of the median values are generally favorable for the variants with mixup. The only exceptions are RandMixFor and RotMixFor with $\alpha \in\{0.25,0.40\}$ for MAE.

Influence of $\alpha$. The following approach shows how the $\alpha$ values can affect the performance measures. For a given dataset, method and performance measure, the values of the measure were calculated for $\alpha=0.1,0.25,0.4$ and then scaled to the interval $[0,1]$. Then, a parabola 
Table 2: Results for RMSE. The best result for each dataset is highlighted with a yellow background.

\begin{tabular}{|c|c|c|c|c|c|c|c|c|c|c|c|c|c|c|}
\hline 2dplanes & 1.109 & 1.112 & 1.112 & 1.112 & 1.112 & 1.112 & 1.112 & 1.09 & 1.043 & 1.04 & 1.039 & 1.043 & 1.04 & 1.04 \\
\hline abalone & 2.193 & 2.189 & 2.187 & 2.179 & 2.181 & 2.179 & 2.173 & 2.111 & 2.114 & 2.113 & 2.113 & 2.115 & 2.114 & 2.115 \\
\hline ailerons & $1.86 \mathrm{e}-04$ & $1.86 \mathrm{e}-04$ & $1.86 \mathrm{e}-04$ & $1.86 \mathrm{e}-04$ & $1.85 \mathrm{e}-04$ & $1.86 \mathrm{e}-04$ & $1.86 \mathrm{e}-04$ & $1.73 \mathrm{e}-04$ & $1.71 \mathrm{e}-04$ & $1.72 \mathrm{e}-04$ & $1.72 \mathrm{e}-04$ & $1.71 \mathrm{e}-04$ & $1.71 \mathrm{e}-04$ & $1.72 \mathrm{e}-04$ \\
\hline auto93 & 6.297 & 6.298 & 6.325 & 6.306 & 6.353 & 6.287 & 6.286 & 6.015 & 5.965 & 5.948 & 5.903 & 5.927 & 5.97 & 5.921 \\
\hline auto-horse & 16.65 & 16.12 & 16.3 & 16.31 & 16.55 & 16.49 & 16.68 & 14.06 & 14.22 & 14.3 & 14.3 & 14.1 & 14.1 & 13.98 \\
\hline auto-mpg & 2.933 & 2.943 & 2.906 & 2.929 & 2.906 & 2.921 & 2.883 & 2.819 & 2.81 & 2.819 & 2.82 & 2.813 & 2.827 & 2.825 \\
\hline auto-price & 2481 & 2465 & 2442 & 2444 & 2474 & 2508 & 2492 & 2487 & 2423 & 2462 & 2447 & 2423 & 2449 & 2451 \\
\hline bank-32nh & 0.08835 & 0.08845 & 0.08847 & 0.08859 & 0.08866 & 0.08888 & 0.08886 & 0.08542 & 0.08484 & 0.0851 & 0.08506 & 0.0849 & 0.08502 & 0.08507 \\
\hline bank-8FM & 0.03268 & 0.0325 & 0.03254 & 0.03256 & 0.03266 & 0.03266 & 0.03273 & 0.03286 & 0.03274 & 0.03281 & 0.03279 & 0.03267 & 0.03265 & 0.03284 \\
\hline baskball & 0.09483 & 0.09551 & 0.09505 & 0.0948 & 0.09368 & 0.09463 & 0.09384 & 0.09327 & 0.09367 & 0.09264 & 0.09313 & 0.09296 & 0.09319 & 0.09287 \\
\hline bodyfat & 2.496 & 2.417 & 2.396 & 2.418 & 2.517 & 2.492 & 2.502 & 2.133 & 2.101 & 2.068 & 2.08 & 2.074 & 2.065 & 2.102 \\
\hline bolts & 13.58 & 12.89 & 13.06 & 13.3 & 13.17 & 13.37 & 13.12 & 13.82 & 13.53 & 13.84 & 14.04 & 13.55 & 13.73 & 13.8 \\
\hline breast-tumor & 10.87 & 10.98 & 10.89 & 10.84 & 10.79 & 10.74 & 10.7 & 10.53 & 10.61 & 10.58 & 10.52 & 10.68 & 10.63 & 10.64 \\
\hline cal-housing & 50325 & 51196 & 52012 & 52442 & 51624 & 52436 & 52746 & 52983 & 53254 & 53875 & 54056 & 53150 & 53608 & 53927 \\
\hline cholesterol & 52.46 & 52.2 & 52.48 & 52.02 & 52.23 & 51.97 & 52.08 & 51.2 & 51.34 & 51.42 & 51.2 & 51.55 & 51.66 & 51.31 \\
\hline cleveland & 0.9146 & 0.9116 & 0.9028 & 0.9014 & 0.904 & 0.9087 & 0.9083 & 0.8903 & 0.8877 & 0.8903 & 0.8854 & 0.8891 & 0.8931 & 0.8877 \\
\hline cloud & 0.5715 & 0.5643 & 0.5722 & 0.5681 & 0.5764 & 0.5721 & 0.5649 & 0.6 & 0.5915 & 0.5873 & 0.5877 & 0.5921 & 0.5913 & 0.5909 \\
\hline сpu & 57.91 & 54.71 & 54.65 & 54.35 & 57.5 & 58.79 & 58.42 & 62.19 & 59.04 & 57.97 & 59.7 & 56.69 & 58.15 & 59.56 \\
\hline cpu-act & 2.562 & 2.541 & 2.553 & 2.551 & 2.562 & 2.561 & 2.566 & 2.519 & 2.551 & 2.552 & 2.561 & 2.545 & 2.564 & 2.563 \\
\hline cpu-small & 2.926 & 2.873 & 2.88 & 2.884 & 2.887 & 2.888 & 2.884 & 2.928 & 2.959 & 2.968 & 2.966 & 2.951 & 2.96 & 2.961 \\
\hline delta-ailerons & $1.69 \mathrm{e}-04$ & $1.67 \mathrm{e}-04$ & $1.67 \mathrm{e}-04$ & $1.67 \mathrm{e}-04$ & $1.66 \mathrm{e}-04$ & $1.67 \mathrm{e}-04$ & $1.67 \mathrm{e}-04$ & $1.70 \mathrm{e}-04$ & $1.68 \mathrm{e}-04$ & $1.68 \mathrm{e}-04$ & $1.68 \mathrm{e}-04$ & $1.68 \mathrm{e}-04$ & $1.68 \mathrm{e}-04$ & $1.68 \mathrm{e}-04$ \\
\hline delta-elevators & $1.46 \mathrm{e}-03$ & $1.46 \mathrm{e}-03$ & $1.46 \mathrm{e}-03$ & $1.46 \mathrm{e}-03$ & $1.46 \mathrm{e}-03$ & $1.46 \mathrm{e}-03$ & $1.45 \mathrm{e}-03$ & $1.43 \mathrm{e}-03$ & $1.43 \mathrm{e}-03$ & $1.43 \mathrm{e}-03$ & $1.43 \mathrm{e}-03$ & $1.43 \mathrm{e}-03$ & $1.43 \mathrm{e}-03$ & $1.43 \mathrm{e}-03$ \\
\hline detroit & 46.99 & 44.51 & 45.65 & 44.77 & 46.24 & 46.84 & 46.62 & 70.75 & 52.58 & 53.31 & 52.66 & 50.55 & 50.15 & 50.69 \\
\hline diabetes-numeric & 0.6433 & 0.6378 & 0.6305 & 0.6266 & 0.6328 & 0.6302 & 0.6297 & 0.6759 & 0.6752 & 0.6723 & 0.6666 & 0.6725 & 0.6655 & 0.6659 \\
\hline echo-months & 12.16 & 12.04 & 12.01 & 12.07 & 12.11 & 12.14 & 12.23 & 12.03 & 11.98 & 12.09 & 12 & 12.05 & 11.97 & 12 \\
\hline elevators & $3.14 \mathrm{e}-03$ & $3.10 \mathrm{e}-03$ & $3.10 \mathrm{e}-03$ & $3.11 \mathrm{e}-03$ & $3.14 \mathrm{e}-03$ & $3.13 \mathrm{e}-03$ & $3.11 \mathrm{e}-03$ & $2.67 \mathrm{e}-03$ & $2.63 \mathrm{e}-03$ & $2.63 \mathrm{e}-03$ & $2.64 \mathrm{e}-03$ & $2.63 \mathrm{e}-03$ & $2.64 \mathrm{e}-03$ & $2.64 \mathrm{e}-03$ \\
\hline elusage & 16.42 & 16 & 15.84 & 15.67 & 16.14 & 15.77 & 15.8 & 13.41 & 13.47 & 13.27 & 13.36 & 13.29 & 13.3 & 13.16 \\
\hline fishcatch & 85.79 & 85.15 & 83.25 & 82.61 & 86.57 & 83.75 & 83.73 & 95.79 & 78.7 & 80.06 & 80.55 & 78.96 & 80.38 & 79.12 \\
\hline fried & 1.377 & 1.368 & 1.375 & 1.377 & 1.381 & 1.383 & 1.388 & 1.441 & 1.429 & 1.446 & 1.452 & 1.444 & 1.455 & 1.465 \\
\hline fruitfly & 19.29 & 19.35 & 19.21 & 19.17 & 18.89 & 18.81 & 18.64 & 17.58 & 17.89 & 17.81 & 17.77 & 17.87 & 17.95 & 17.76 \\
\hline gascons & 10.75 & 10.35 & 10.68 & 10.83 & 11.09 & 11.4 & 11.9 & 17.02 & 13.4 & 13.3 & 12.66 & 13.53 & 12.91 & 13.09 \\
\hline house- $16 \mathrm{H}$ & 32615 & 32465 & 32604 & 32587 & 32633 & 32778 & 32742 & 34145 & 33763 & 33884 & 33994 & 33737 & 33851 & 33931 \\
\hline house- $8 \mathrm{~L}$ & 29979 & 29917 & 29912 & 29887 & 29887 & 29872 & 29908 & 30593 & 30483 & 30497 & 30520 & 30403 & 30519 & 30520 \\
\hline housing & 3.598 & 3.559 & 3.549 & 3.572 & 3.535 & 3.53 & 3.524 & 3.721 & 3.633 & 3.637 & 3.643 & 3.615 & 3.62 & 3.646 \\
\hline hungarian & 0.3707 & 0.3712 & 0.3692 & 0.3691 & 0.3676 & 0.3672 & 0.3661 & 0.3627 & 0.3612 & 0.3593 & 0.3588 & 0.3631 & 0.3616 & 0.3623 \\
\hline kin8nm & 0.1503 & 0.1487 & 0.1492 & 0.1493 & 0.1501 & 0.1502 & 0.1504 & 0.1291 & 0.1279 & 0.1291 & 0.1298 & 0.128 & 0.1287 & 0.13 \\
\hline longley & 1325 & 1331 & 1317 & 1323 & 1400 & 1366 & 1361 & 1730 & 1600 & 1574 & 1580 & 1559 & 1625 & 1582 \\
\hline lowbwt & 462.9 & 468.5 & 466.5 & 469.2 & 460.1 & 468.6 & 466 & 457.2 & 461.7 & 461.6 & 460 & 462.8 & 458.2 & 461.6 \\
\hline machine-cpu & 65.06 & 64.39 & 64.63 & 65.17 & 63.81 & 64.02 & 65.36 & 77.62 & 72.64 & 72.66 & 72.8 & 72.19 & 73.48 & 72.97 \\
\hline mbagrade & 0.3755 & 0.3794 & 0.3761 & 0.3726 & 0.3673 & 0.3649 & 0.3634 & 0.3305 & 0.3403 & 0.3369 & 0.3317 & 0.3401 & 0.337 & 0.3327 \\
\hline meta & 748.4 & 747.8 & 741.1 & 743.8 & 744.3 & 745.5 & 741.5 & 725.3 & 730.6 & 733.2 & 731.9 & 735.7 & 739.6 & 737.9 \\
\hline $\mathrm{mv}$ & 0.2727 & 0.2719 & 0.3235 & 0.3686 & 0.3133 & 0.3958 & 0.4519 & 0.2231 & 0.2979 & 0.3672 & 0.4067 & 0.2394 & 0.2545 & 0.2626 \\
\hline $\mathrm{pbc}$ & 920.2 & 922.6 & 918.7 & 918.1 & 918.7 & 921.2 & 920.3 & 880.5 & 879.4 & 880.5 & 881.3 & 882.1 & 879.8 & 880.7 \\
\hline pharynx & 357.4 & 357.3 & 358.8 & 357.2 & 360.2 & 360.6 & 360.7 & 313.2 & 311.1 & 312.5 & 311.4 & 311.3 & 312.3 & 314 \\
\hline pol & 7.315 & 7.562 & 7.814 & 7.947 & 7.839 & 8.097 & 8.27 & 5.268 & 5.573 & 5.996 & 6.23 & 5.693 & 6.106 & 6.364 \\
\hline pollution & 48.92 & 48.23 & 48.55 & 48.72 & 49.25 & 49.26 & 49.27 & 46.84 & 47.28 & 47.6 & 47.27 & 46.85 & 47.29 & 47.64 \\
\hline puma32H & 0.01687 & 0.01728 & 0.01743 & 0.01754 & 0.01761 & 0.01808 & 0.0183 & 0.01313 & 0.01359 & 0.01383 & 0.01393 & 0.01364 & 0.01385 & 0.01406 \\
\hline puma $8 \mathrm{NH}$ & 3.253 & 3.257 & 3.27 & 3.275 & 3.266 & 3.284 & 3.293 & 3.278 & 3.291 & 3.313 & 3.319 & 3.289 & 3.305 & 3.316 \\
\hline pw-linear & 2.09 & 2.064 & 2.07 & 2.077 & 2.069 & 2.108 & 2.087 & 1.881 & 1.883 & 1.896 & 1.896 & 1.896 & 1.897 & 1.907 \\
\hline pyrim & 0.1004 & 0.1008 & 0.1001 & 0.09966 & 0.09672 & 0.09815 & 0.09838 & 0.1198 & 0.1056 & 0.1048 & 0.1066 & 0.1067 & 0.1072 & 0.1075 \\
\hline quake & 0.1981 & 0.1985 & 0.1972 & 0.1972 & 0.1967 & 0.1965 & 0.1963 & 0.1904 & 0.1933 & 0.1914 & 0.1909 & 0.1931 & 0.1915 & 0.1908 \\
\hline schlvote & 1159316 & 1178402 & 1176517 & 1175270 & 1162099 & 1155803 & 1149687 & 1123137 & 1082854 & 1104934 & 1091608 & 1096670 & 1089156 & 1091314 \\
\hline sensory & 0.7298 & 0.7321 & 0.733 & 0.7299 & 0.73 & 0.7327 & 0.7313 & 0.7228 & 0.7234 & 0.7221 & 0.7244 & 0.7217 & 0.7232 & 0.7224 \\
\hline servo & 0.7638 & 0.7533 & 0.7802 & 0.782 & 0.7707 & 0.7813 & 0.7912 & 0.7451 & 0.7746 & 0.7693 & 0.7716 & 0.7407 & 0.761 & 0.7426 \\
\hline sleep & 3.658 & 3.695 & 3.667 & 3.634 & 3.614 & 3.624 & 3.614 & 3.439 & 3.42 & 3.433 & 3.431 & 3.411 & 3.446 & 3.426 \\
\hline stock & 0.9121 & 0.8822 & 0.887 & 0.8879 & 0.9033 & 0.9098 & 0.9033 & 0.8486 & 0.8422 & 0.8474 & 0.8506 & 0.8437 & 0.8488 & 0.8549 \\
\hline strike & 537.2 & 544.3 & 538.7 & 537 & 534.7 & 534.7 & 534.2 & 516.2 & 513.7 & 510 & 513.2 & 511 & 513 & 510.6 \\
\hline triazines & 0.1355 & 0.1348 & 0.1352 & 0.1346 & 0.1351 & 0.1343 & 0.135 & 0.1388 & 0.1363 & 0.1369 & 0.1371 & 0.1361 & 0.1367 & 0.1364 \\
\hline veteran & 149.8 & 150.8 & 149.1 & 149.1 & 149.5 & 148.8 & 149.9 & 145.8 & 145.4 & 145.3 & 144.4 & 148.4 & 146.3 & 146.5 \\
\hline vineyard & 2.622 & 2.599 & 2.607 & 2.607 & 2.601 & 2.607 & 2.622 & 3.021 & 2.906 & 2.928 & 2.918 & 2.935 & 2.919 & 2.937 \\
\hline wisconsin & 33.26 & 33.48 & 33.49 & 33.25 & 33.31 & 33.07 & 33.15 & 33.01 & 32.9 & 32.88 & 32.88 & 32.91 & 32.91 & 32.84 \\
\hline
\end{tabular}


Table 3: Results for MAE. The best result for each dataset is highlighted with a yellow background.

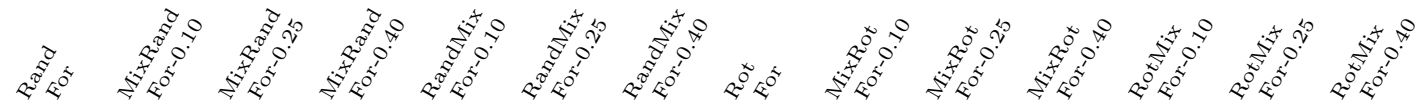

\begin{tabular}{|c|c|c|c|c|c|c|c|c|c|c|c|c|c|c|}
\hline 2dplanes & 8819 & 0.885 & 0.8845 & 0.8845 & 0.8842 & 0.8846 & 0.8846 & 0.8682 & 0.8313 & 0.8291 & 0.8278 & 0.8313 & 0.829 & 0.8287 \\
\hline abalone & .553 & 1.549 & 1.545 & 1.54 & 1.54 & 1.539 & 1.533 & 1.485 & 1.489 & 1.487 & 1.485 & 1.487 & 1.489 & 1.487 \\
\hline ailerons & $1.37 \mathrm{e}-04$ & $1.37 \mathrm{e}-04$ & $1.37 \mathrm{e}-04$ & $1.37 \mathrm{e}-04$ & $1.37 \mathrm{e}-04$ & $1.37 \mathrm{e}-04$ & $1.38 \mathrm{e}-04$ & $1.28 \mathrm{e}-04$ & $1.25 \mathrm{e}-04$ & $1.26 \mathrm{e}-04$ & $1.27 \mathrm{e}-04$ & $1.26 \mathrm{e}-04$ & $1.26 \mathrm{e}-04$ & $1.27 \mathrm{e}-04$ \\
\hline auto93 & 4.417 & 4.323 & 4.333 & 4.341 & 4.419 & 4.366 & 4.371 & 3.949 & 3.917 & 3.877 & 3.873 & 3.86 & 3.933 & 3.885 \\
\hline auto-horse & 766 & 9.292 & 9.462 & 9.442 & 9.65 & 688 & 9.862 & 7.46 & 7.528 & 7.657 & 7.607 & 7.475 & .482 & 7.38 \\
\hline auto-mpg & 2.117 & 2.118 & 2.098 & 2.111 & 2.095 & 2.115 & 2.098 & 2.004 & 1.999 & 2.009 & 2.016 & 2.009 & 2.011 & 2.024 \\
\hline auto-price & 1552 & 1551 & 1541 & 1541 & 1560 & 1582 & 1561 & 1574 & 1525 & 1549 & 1543 & 1532 & 1550 & 1545 \\
\hline bank-32nh & .06171 & 0.06221 & 0.0628 & 0.06312 & 0.06301 & 0.06379 & 0.06399 & 0.05811 & 0.05796 & 0.05855 & 0.05882 & 0.05796 & 0.05853 & 0.05887 \\
\hline bank-8FM & 02353 & 0.02346 & 0.0235 & 0.02355 & 0.02358 & 0.02366 & 0.02376 & 0.02452 & 0.0245 & 0.02466 & 0.02469 & 0.02446 & 0.02452 & 0.02476 \\
\hline baskball & 0.07279 & 0.07399 & 0.07354 & 0.07318 & 0.07219 & 0.07311 & 0.07251 & 0.07306 & 0.07363 & 0.07244 & 0.07299 & 0.07283 & 0.07312 & 0.07274 \\
\hline bodyfat & 1.752 & 1.695 & 1.676 & 1.692 & 1.774 & 1.754 & 1.751 & 1.383 & 1.364 & 1.336 & 1.333 & 1.341 & 1.326 & 1.362 \\
\hline bolts & 10.05 & 9.534 & 9.734 & 9.927 & 9.883 & 10.04 & 9.974 & 10.04 & 9.93 & 10.15 & 10.46 & 9.797 & 10.17 & 10.13 \\
\hline breast-tumor & 8.629 & 8.695 & 8.638 & 8.613 & 8.57 & 8.522 & 8.504 & 8.383 & 8.437 & 8.425 & 8.357 & 8.501 & 8.468 & 8.467 \\
\hline cal-housing & 33431 & 34315 & 35085 & 35529 & 34796 & 35579 & 35942 & 35978 & 36258 & 36991 & 37249 & 36159 & 36764 & 37149 \\
\hline cholester & 40.03 & 39.56 & 39.82 & 39.54 & 39.75 & 39.66 & 39.54 & 39.01 & 39.02 & 39.16 & 38.97 & 39.18 & 39.37 & 39.13 \\
\hline cleveland & 0.6794 & 0.6756 & 0.6735 & 0.6743 & 0.677 & 0.6817 & 0.6834 & 0.6463 & 0.6449 & 0.6474 & 0.6478 & 0.6453 & 0.6493 & 0.649 \\
\hline cloud & 0.3381 & 0.3314 & 0.3338 & 0.3324 & 0.3356 & 0.3347 & 0.3301 & 0.3549 & 0.35 & 0.3459 & 0.3492 & 0.3493 & 0.3512 & 0.3509 \\
\hline cpu & 20.25 & 19.16 & 19.26 & 18.86 & 19.37 & 19.7 & 19.69 & 20.08 & 17.05 & 16.87 & 17.66 & 16.38 & 17.01 & 17.6 \\
\hline cpu-act & 1.8 & 1.808 & 1.826 & 1.826 & 1.83 & 1.835 & 1.836 & 1.765 & 1.8 & 1.812 & 1.816 & 1.8 & 1.818 & 1.819 \\
\hline cpu-small & 2.04 & 2.035 & 2.047 & 2.048 & 2.05 & 2.05 & 2.053 & 2.076 & 2.112 & 2.131 & 2.133 & 2.108 & 2.126 & 2.133 \\
\hline delta-ailer & $1.17 \mathrm{e}-04$ & $1.16 \mathrm{e}-04$ & $1.15 \mathrm{e}-04$ & $1.16 \mathrm{e}-04$ & $1.15 \mathrm{e}-04$ & $1.15 \mathrm{e}-04$ & $1.16 \mathrm{e}-04$ & $1.17 \mathrm{e}-04$ & $1.16 \mathrm{e}-04$ & $1.16 \mathrm{e}-04$ & $1.16 \mathrm{e}-04$ & $1.16 \mathrm{e}-04$ & $1.16 \mathrm{e}-04$ & $1.16 \mathrm{e}-04$ \\
\hline delta-ele & $1.09 \mathrm{e}-03$ & $1.09 \mathrm{e}-03$ & $1.09 \mathrm{e}-03$ & $1.09 \mathrm{e}-03$ & $1.09 \mathrm{e}-03$ & $1.09 \mathrm{e}-03$ & $1.09 \mathrm{e}-03$ & $1.07 \mathrm{e}-03$ & $1.07 \mathrm{e}-03$ & $1.07 \mathrm{e}-03$ & $1.07 \mathrm{e}-03$ & $1.07 \mathrm{e}-03$ & $1.07 \mathrm{e}-03$ & $1.07 \mathrm{e}-03$ \\
\hline detroit & 35.71 & 34.8 & 35.55 & 34.85 & 35.13 & 35.66 & 35.76 & 55.09 & 40.13 & 40.63 & 40 & 38.04 & 37.85 & 38.36 \\
\hline diabetes-r & 0.5119 & 0.5078 & 0.5014 & 0.4945 & 0.5035 & 0.5007 & 0.4996 & 0.5487 & 0.5376 & 0.5333 & 0.529 & 0.5369 & 0.5299 & 0.528 \\
\hline echo-mont & 9.625 & 9.436 & 9.516 & 9.595 & 9.675 & 9.755 & 9.826 & 9.565 & 9.512 & 9.674 & 9.591 & 9.563 & 9.529 & 9.547 \\
\hline elev & $2.12 \mathrm{e}-03$ & $2.09 \mathrm{e}-03$ & $2.09 \mathrm{e}-03$ & $2.10 \mathrm{e}-03$ & $2.12 \mathrm{e}-03$ & $2.11 \mathrm{e}-03$ & $2.10 \mathrm{e}-03$ & $1.84 \mathrm{e}-03$ & $1.81 \mathrm{e}-03$ & $1.82 \mathrm{e}-03$ & $1.82 \mathrm{e}-03$ & $1.81 \mathrm{e}-03$ & $1.82 \mathrm{e}-03$ & $1.82 \mathrm{e}-03$ \\
\hline elusage & 12.61 & 12.21 & 12.1 & 11.92 & 12.39 & 12.2 & 12.2 & 9.894 & 10.04 & 9.919 & 9.951 & 9.952 & 9.903 & 9.817 \\
\hline fishcatch & 53.23 & 52.17 & 51.15 & 51.17 & 52.92 & 52 & 52.08 & 55.35 & 47 & 48.8 & 49.15 & 47.74 & 48.63 & 48.27 \\
\hline ried & 1.087 & 1.08 & 1.085 & 1.086 & 1.089 & 1.092 & 1.096 & 1.136 & 28 & 1.142 & 47 & 1.139 & 49 & 1.158 \\
\hline fruitfly & 14.49 & 14.53 & 14.44 & 14.45 & 14.18 & 14.1 & 13.99 & 12.99 & 13 & 28 & 13.18 & 13.32 & 38 & 13.22 \\
\hline gascons & 8.42 & 8.032 & 8.331 & 8.48 & 8.635 & 8.775 & 9.181 & 13.29 & 10 & 10.25 & 9.755 & 10.39 & 10.04 & 10.05 \\
\hline house- $16 \mathrm{H}$ & 16388 & 16326 & 16407 & 16424 & 16418 & 16471 & 16503 & 17583 & 17 & 17520 & 17624 & 17315 & 17 & 17593 \\
\hline house-8L & 15814 & 15784 & 15787 & 15790 & 15781 & 15807 & 15850 & 16417 & 16263 & 16349 & 16409 & 16233 & 16354 & 16408 \\
\hline housing & 2.389 & 2.37 & 2.377 & 2.372 & 2.368 & 2.379 & 2.373 & 2.415 & 71 & 72 & 2.379 & 2.352 & 2.361 & 2.373 \\
\hline hung & 0.2663 & 0.267 & 0.2674 & 0.2703 & 0.2 & 0.2721 & 0.274 & 0.2507 & 0.251 & 0.254 & 0.2555 & 0.251 & 0.252 & 0.2549 \\
\hline $\sin 8 \mathrm{~nm}$ & 1196 & $0.11 \varepsilon$ & 0.1194 & 0.1196 & 0.1202 & 0.1205 & 0.121 & 0.1022 & 0.1015 & 0.1028 & 0.1036 & 0.1015 & 0.1025 & 0.1037 \\
\hline ongley & 1145 & 1127 & 1116 & 1106 & 1187 & 1156 & 1149 & 1428 & 5 & 1299 & 1296 & 1281 & 1328 & 1299 \\
\hline owbwt & 363.8 & 365.6 & 362.9 & 36 & 359.7 & 364 & 361 & 362.3 & .5 & 5 & 360.3 & 363.7 & 360.4 & 362.1 \\
\hline nac & 30.76 & 30.36 & 30.57 & 30.81 & 30.24 & 30.69 & 30.8 & 35.33 & 33.06 & 33.2 & 32.88 & 32.91 & 33.05 & 33.13 \\
\hline nbagrad & 0.2841 & 0.288 & 0.2861 & 0.2835 & 0.2787 & 0.2755 & 0.2758 & 0.2521 & 0.2544 & 0.253 & 0.2503 & 0.2546 & 0.2536 & 0.2509 \\
\hline neta & 145.2 & 146 & 144.9 & 146.6 & 145.2 & 146.8 & 146.4 & 148.5 & 147 & 147.2 & 148.9 & 148.1 & 149.7 & 149.1 \\
\hline $\mathrm{nv}$ & 0.18 & 0.1774 & 0.2155 & 0.2444 & 0.208 & 0.2622 & 0.2968 & 0.1515 & 0.2158 & 0.2759 & 0.3061 & 0.1661 & 0.1814 & 0.1896 \\
\hline $\mathrm{pbc}$ & 717.7 & 720.2 & 720.4 & 720.2 & 719.3 & 724.5 & 722.3 & 695.2 & 695.8 & 697.7 & 697.3 & 696.5 & 694.3 & 698.6 \\
\hline phar & 278.5 & 279 & 281 & 278.8 & 282.2 & 282.5 & 282.6 & 234.2 & 232.2 & 232.6 & 231.8 & 232.3 & 233.3 & 234.2 \\
\hline pol & 4.134 & 4.391 & 4.64 & 4.766 & 4.632 & 4.864 & 5.032 & 2.646 & 2.994 & 3.398 & 3.621 & 3.068 & 3.465 & 3.687 \\
\hline pollution & 37.37 & 37.02 & 37.22 & 37.12 & 37.66 & 37.65 & 37.81 & 35.39 & 36.1 & 36.09 & 35.9 & 35.6 & 6.01 & 36.47 \\
\hline puma32H & 0.01299 & 0.01328 & 0.01342 & 0.0135 & 0.01355 & 0.01392 & 0.01407 & 0.01047 & 0.01085 & 0.01106 & 0.01114 & 0.01089 & 0.01107 & 0.01124 \\
\hline puma $8 \mathrm{NH}$ & 2.528 & 2.55 & 2.572 & 2.587 & 2.572 & 2.6 & 2.618 & 2.589 & 2.61 & 2.642 & 2.653 & 2.608 & 2.634 & 2.652 \\
\hline pw-linear & 1.635 & 1.612 & 1.609 & 1.618 & 1.617 & 1.641 & 1.628 & 1.449 & 1.439 & 1.46 & 1.45 & 1.452 & 1.45 & 1.464 \\
\hline pyrim & 0.06158 & 0.062 & 0.06161 & 0.06177 & 0.06099 & 0.0614 & 0.06139 & 0.07847 & 0.06655 & 0.06653 & 0.06726 & 0.06728 & 0.06793 & 0.06794 \\
\hline quake & 0.1549 & 0.155 & 0.1541 & 0.1541 & 0.154 & 0.1539 & 0.1537 & 0.1494 & 0.151 & 0.15 & 0.1497 & 0.1509 & 0.15 & 0.1497 \\
\hline schlvote & 639146 & 659770 & 667857 & 664392 & 653918 & 656491 & 665144 & 677601 & 652816 & 666136 & 657503 & 656814 & 651958 & 656596 \\
\hline sensory & 0.5857 & 0.5864 & 0.5849 & 0.5829 & 0.5842 & 0.586 & 0.5856 & 0.5794 & 0.5792 & 0.5777 & 0.58 & 0.5793 & 0.5787 & 0.5785 \\
\hline servo & 0.4635 & 0.4578 & 0.4801 & 0.4866 & 0.4772 & 0.4908 & 0.5022 & 0.4327 & 0.4642 & 0.4693 & 0.4779 & 0.4454 & 0.4645 & 0.4567 \\
\hline sleep & 2.937 & 2.957 & 2.955 & 2.941 & 2.907 & 2.91 & 2.906 & 2.688 & 2.679 & 2.682 & 2.683 & 2.664 & 2.699 & 2.657 \\
\hline stoc & 0.669 & 0.6516 & 0.6547 & 0.657 & 0.6668 & 0.6717 & 0.6699 & 0.6361 & 0.6344 & 0.6371 & 0.6385 & 0.6338 & 0.6388 & 0.6444 \\
\hline strike & 211.9 & 214.7 & 212.9 & 212.6 & 212.9 & 212.3 & 212.2 & 245.9 & 230.4 & 231.2 & 237 & 229.3 & 235.3 & 236.7 \\
\hline triazines & 0.09521 & 0.09518 & 0.09548 & 0.09497 & 0.09498 & 0.09503 & 0.09517 & 0.09955 & 0.09742 & 0.09805 & 0.09784 & 0.0971 & 0.09773 & 0.09744 \\
\hline veteran & 95.34 & 95.48 & 94.23 & 93.92 & 94.44 & 93.98 & 94.81 & 90.53 & 89.82 & 90.37 & 90.27 & 91.56 & 91.09 & 91.97 \\
\hline & 1.95 & 1.949 & 1.961 & 1.961 & 1.932 & 1.956 & 1.961 & 2.311 & 2.199 & 2.224 & 2.216 & 2.22 & 2.212 & 2.219 \\
\hline Visconsin & 28.02 & 28.14 & 28.27 & 28.04 & 28.07 & 27.88 & 27.98 & 27.75 & 27.68 & 27.69 & 27.66 & 27.81 & 27.76 & 27.66 \\
\hline
\end{tabular}


Table 4: Results for correlation. The best result for each dataset is highlighted with a yellow background.

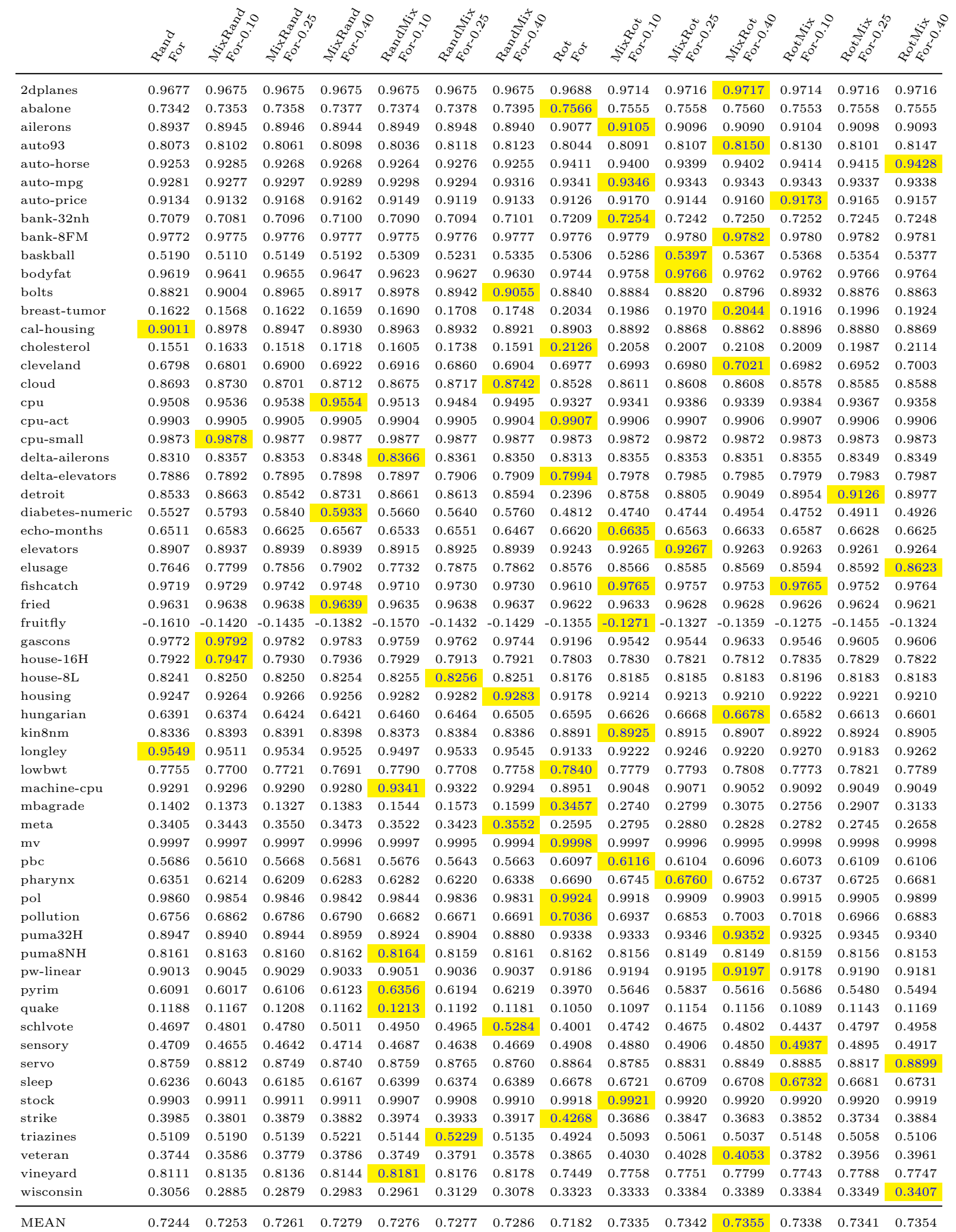


Table 5: Comparisons of Random Forest variants. Each cell shows the number of datasets where the column method is better than the row method.

(a) RMSE

\begin{tabular}{|c|c|c|c|c|c|c|c|c|}
\hline RandFor & & 36 & 39 & 45 & 39 & 39 & 32 & 230 \\
\hline MixRandFor-0.10 & 24 & & 29 & 30 & 27 & 28 & 28 & 166 \\
\hline MixRandFor-0.25 & 21 & 30 & & 31 & 29 & 25 & 25 & 161 \\
\hline MixRandFor- 0.40 & 15 & 29 & 28 & & 25 & 25 & 20 & 142 \\
\hline RandMixFor-0.10 & 22 & 34 & 31 & 36 & & 25 & 27 & 175 \\
\hline RandMixFor-0.25 & 21 & 31 & 34 & 34 & 36 & & 33 & 189 \\
\hline RandMixFor-0.40 & 28 & 31 & 34 & 39 & 34 & 26 & & 192 \\
\hline Total & 131 & 191 & 195 & 215 & 190 & 168 & 165 & \\
\hline
\end{tabular}

(b) MAE

\begin{tabular}{llllllll|l} 
& 36 & 33 & 33 & 27 & 28 & 192 \\
\hline RandFor & & 35 & 36 & 32 & 28 & 22 & 22 & 148 \\
MixRandFor-0.10 & 25 & & 25 & 28 & 26 & 22 \\
MixRandFor-0.25 & 24 & 34 & & 25 & 29 & 17 & 19 & 148 \\
MixRandFor-0.40 & 27 & 31 & 35 & & 28 & 16 & 18 & 155 \\
RandMixFor-0.10 & 27 & 34 & 30 & 31 & & 19 & 18 & 159 \\
RandMixFor-0.25 & 33 & 38 & 42 & 44 & 40 & & 27 & 224 \\
RandMixFor-0.40 & 33 & 38 & 42 & 42 & 43 & 33 & & 231 \\
\hline Total & 169 & 210 & 210 & 203 & 199 & 134 & 132 &
\end{tabular}

(c) Correlation

\begin{tabular}{|c|c|c|c|c|c|c|c|c|}
\hline RandFor & & 40 & 40 & 46 & 44 & 44 & 43 & 257 \\
\hline MixRandFor-0.10 & 21 & & 33 & 39 & 31 & 33 & 33 & 190 \\
\hline MixRandFor-0.25 & 21 & 28 & & 40 & 34 & 31 & 35 & 189 \\
\hline MixRandFor-0.40 & 15 & 22 & 21 & & 24 & 30 & 30 & 142 \\
\hline RandMixFor- 0.10 & 17 & 30 & 27 & 36 & & 30 & 31 & 171 \\
\hline RandMixFor-0.25 & 17 & 28 & 30 & 31 & 31 & & 37 & 174 \\
\hline RandMixFor- 0.40 & 18 & 28 & 26 & 31 & 30 & 24 & & 157 \\
\hline Total & 109 & 176 & 177 & 223 & 194 & 192 & 209 & \\
\hline
\end{tabular}


Table 6: Comparisons of Rotation Forest variants. Each cell shows the number of datasets where the column method is better than the row method.

(a) RMSE

\begin{tabular}{|c|c|c|c|c|c|c|c|c|}
\hline RotFor & & 38 & 35 & 36 & 37 & 34 & 36 & 216 \\
\hline MixRotFor-0.10 & 23 & & 24 & 24 & 28 & 22 & 22 & 143 \\
\hline MixRotFor-0.25 & 26 & 36 & & 28 & 36 & 26 & 26 & 178 \\
\hline MixRotFor-0.40 & 25 & 36 & 30 & & 37 & 30 & 26 & 184 \\
\hline RotMixFor-0.10 & 24 & 30 & 24 & 23 & & 20 & 24 & 145 \\
\hline RotMixFor-0.25 & 27 & 37 & 33 & 29 & 39 & & 24 & 189 \\
\hline RotMixFor- 0.40 & 25 & 38 & 33 & 33 & 36 & 35 & & 200 \\
\hline Total & 150 & 215 & 179 & 173 & 213 & 167 & 158 & \\
\hline
\end{tabular}

(b) MAE

\begin{tabular}{llllllll|l} 
& 31 & 31 & 35 & 29 & 32 & 197 \\
\hline RotFor & & 39 & 31 & & \\
MixRotFor-0.10 & 22 & & 20 & 22 & 34 & 20 & 21 & 139 \\
MixRotFor-0.25 & 30 & 40 & & 27 & 38 & 29 & 22 & 186 \\
MixRotFor-0.40 & 30 & 38 & 32 & & 39 & 32 & 23 & 194 \\
RotMixFor-0.10 & 26 & 25 & 21 & 21 & & 19 & 20 & 132 \\
RotMixFor-0.25 & 32 & 40 & 30 & 28 & 40 & & 24 & 194 \\
RotMixFor-0.40 & 29 & 39 & 38 & 36 & 40 & 35 & & 217 \\
\hline Total & 169 & 221 & 172 & 165 & 226 & 164 & 142 &
\end{tabular}

(c) Correlation

\begin{tabular}{|c|c|c|c|c|c|c|c|c|}
\hline RotFor & & 40 & 41 & 41 & 40 & 42 & 43 & 247 \\
\hline MixRotFor-0.10 & 21 & & 30 & 31 & 33 & 30 & 29 & 174 \\
\hline MixRotFor-0.25 & 20 & 31 & & 31 & 35 & 25 & 34 & 176 \\
\hline MixRotFor-0.40 & 20 & 30 & 30 & & 31 & 21 & 29 & 161 \\
\hline RotMixFor-0.10 & 21 & 27 & 26 & 29 & & 23 & 32 & 158 \\
\hline RotMixFor- 0.25 & 19 & 31 & 35 & 40 & 38 & & 32 & 195 \\
\hline RotMixFor-0.40 & 18 & 32 & 27 & 32 & 29 & 29 & & 167 \\
\hline Total & 119 & 191 & 189 & 204 & 206 & 170 & 199 & \\
\hline
\end{tabular}


Figure 5: Boxplots of relative performances. The start and end of the box are the first and third quartiles, the band inside the box is the median. Outliers are not shown.

(a) RMSE
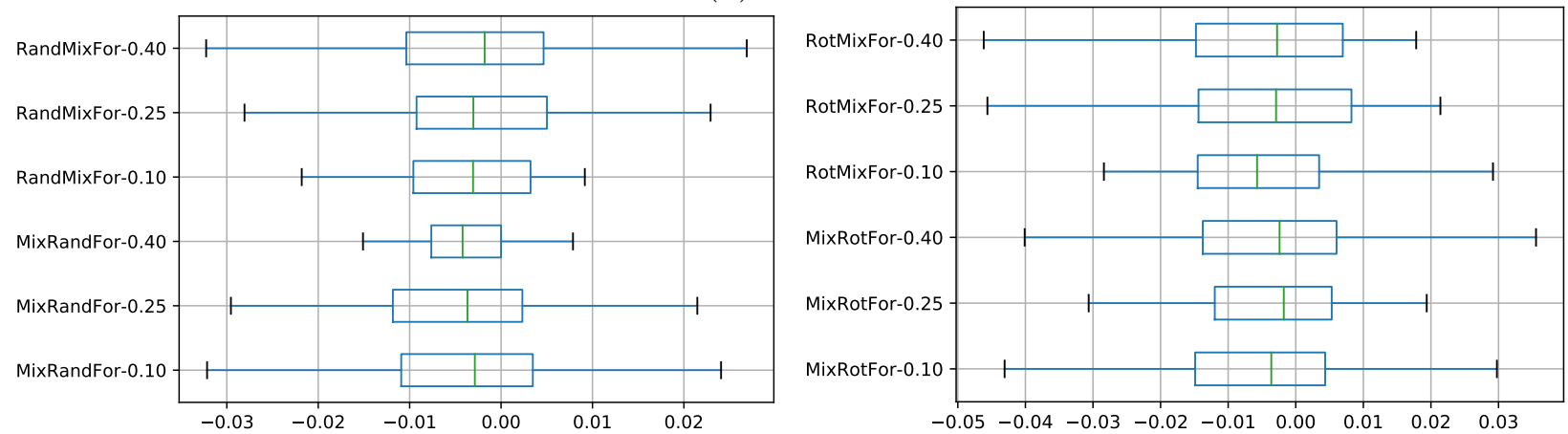

(b) MAE
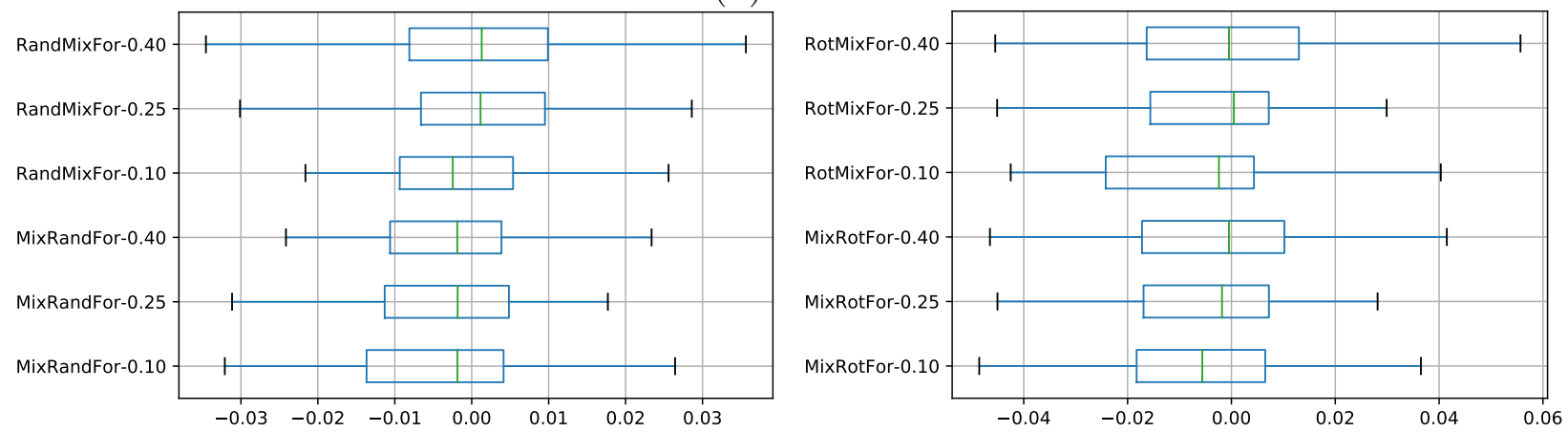

(c) Correlation
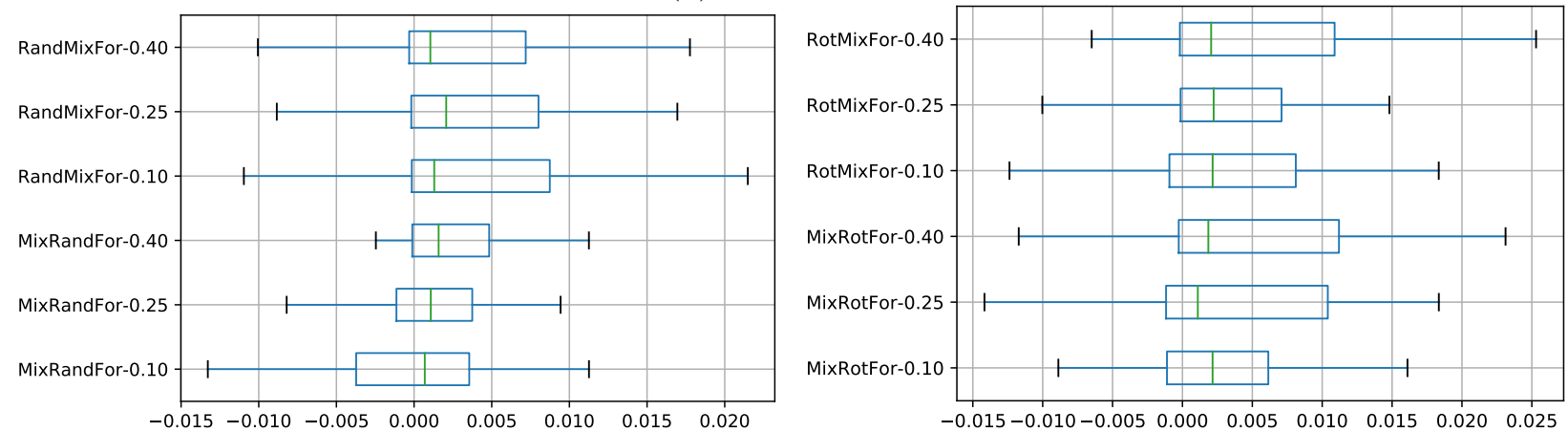

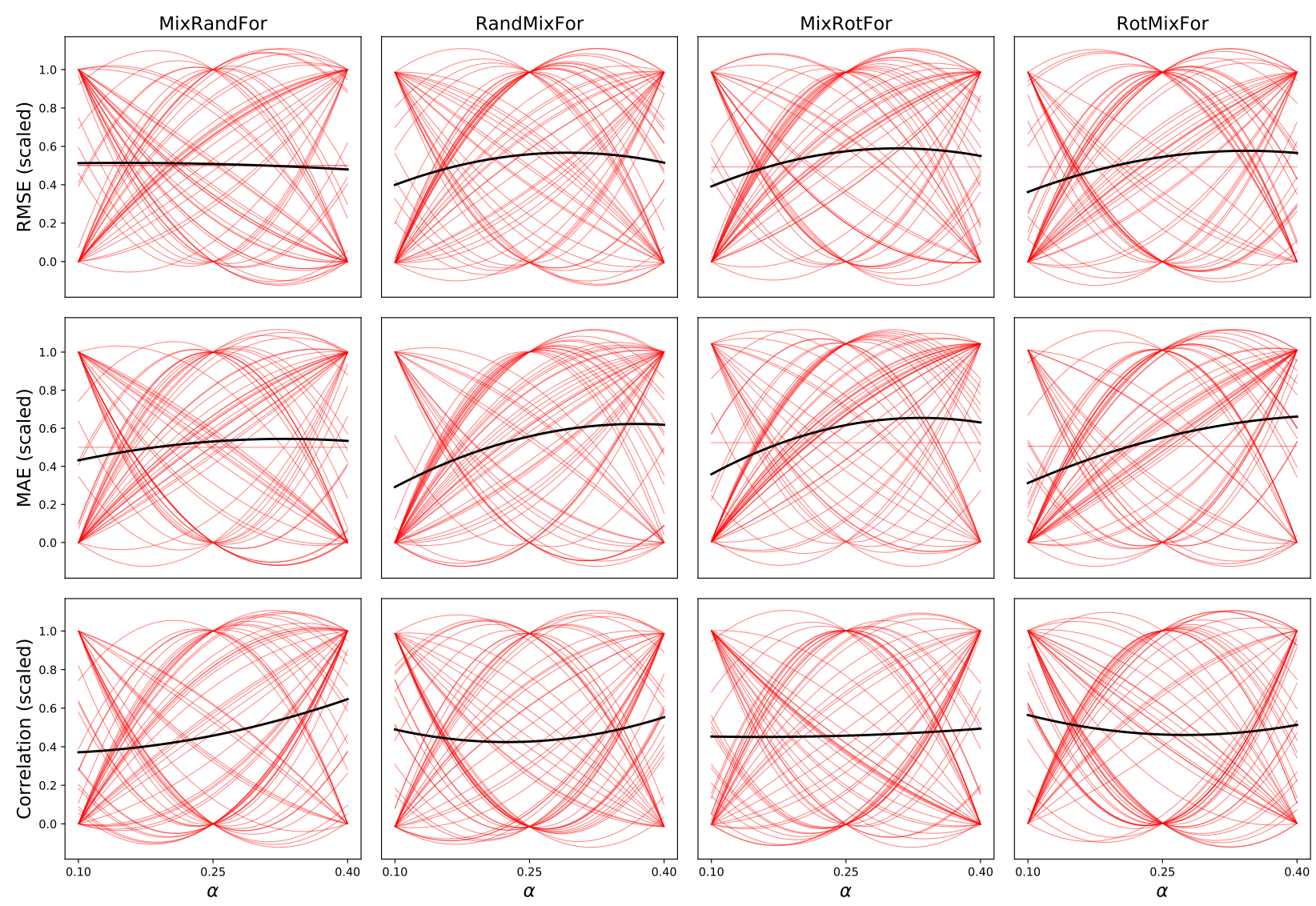

Figure 6: Scaled measures as a function of $\alpha$. Each red parabola corresponds to a single dataset; the black parabola plots the average values.

was fitted to the three points. Figure 6 shows these parabolas, and a final parabola (shown in black) obtained by averaging the scaled values across all the datasets. There is no consistent pattern of the parabolas for the individual datasets, indicating that the optimal value of $\alpha$ depends on the dataset.

Average ranks. Figure 7 shows the average ranks for Random Forest and its variants with mixup. The best method is assigned rank 1, the second is assigned rank 2, and so on. The worst method is assigned rank 7, as we are comparing 7 alternatives for each ensemble method (the original ensemble, MixXXX for three values of $\alpha$, and XXXMix for three values of $\alpha$.) With the aim of evaluating whether some variants are significantly better than the starting method (without mixup), the Bonferroni-Dunn test was performed over the 
(a) RMSE

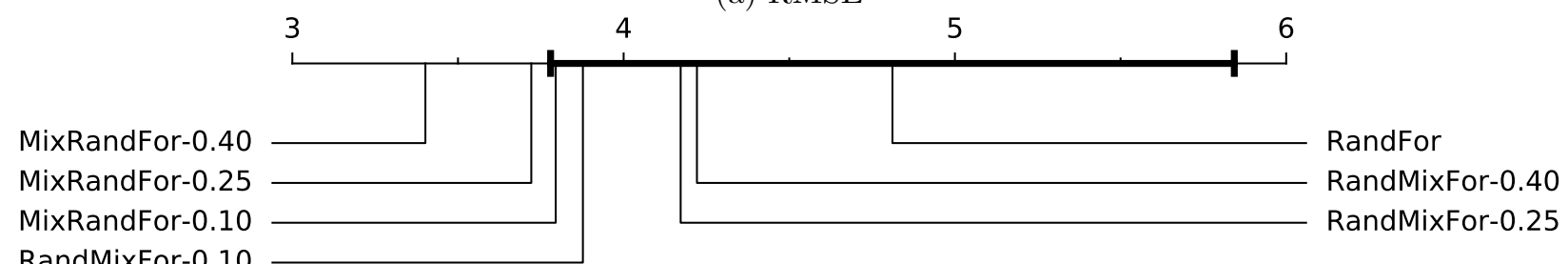

(b) MAE

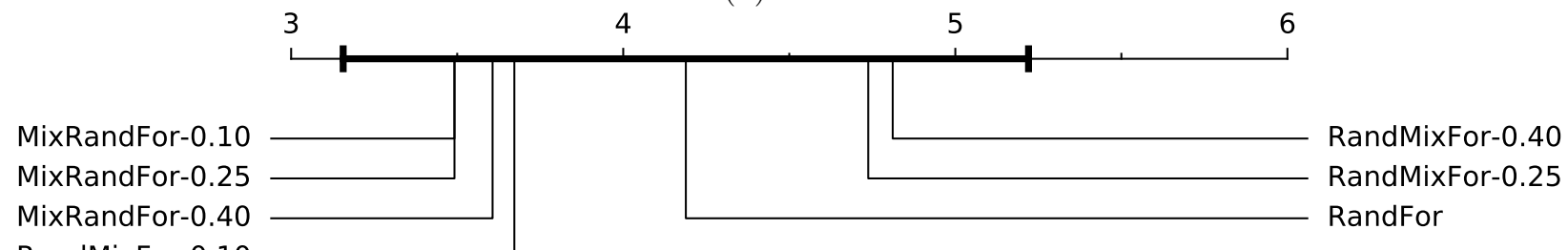

RandMixFor-0.10

(c) Correlation

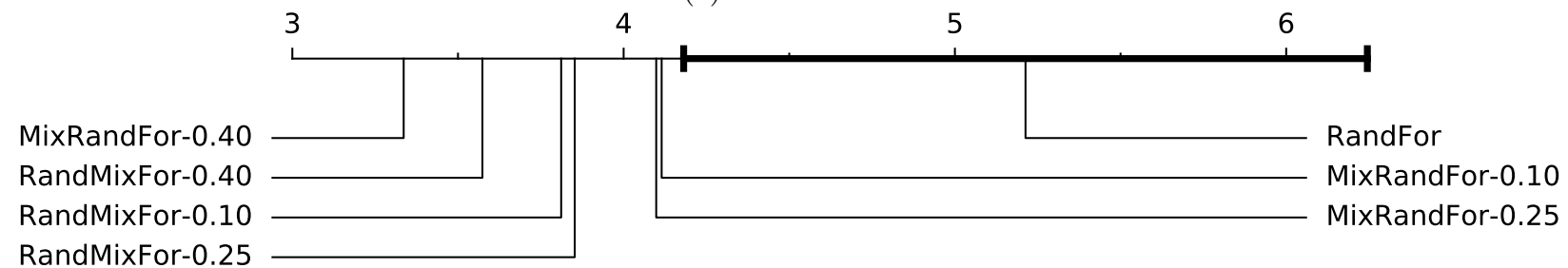

Figure 7: Comparison of Random Forest against variants with Mixup, with the Bonferroni-Dunn test. The marked interval spans the critical value and is centered at the mean rank for Random Forest. Variants with ranks outside the marked interval are significantly different $(p<0.05)$ than Random Forest.

ranks (Demšar, 2006) using Random or Rotation Forest as the control classifier. Random Forest without mixup had the worst average rank for RMSE and correlation. The advantage of mixup for MAE was less clear, as two variants with mixup were worse.

Figure 8 shows the average ranks for Rotation Forest and its mixup variants. In the same way as Random Forest, Rotation Forest without mixup shows the worst average rank for RMSE and correlation. The three variants with mixup were worse for MAE, while the other three were better.

Table 7 shows the average ranks for Random Forest, Rotation Forest, and their variants with mixup. Instead of having two independent ranks, one for Random Forest and the other for Rotation Forest, as with the two previous Figures (7 and 8), these tables show the ranks 

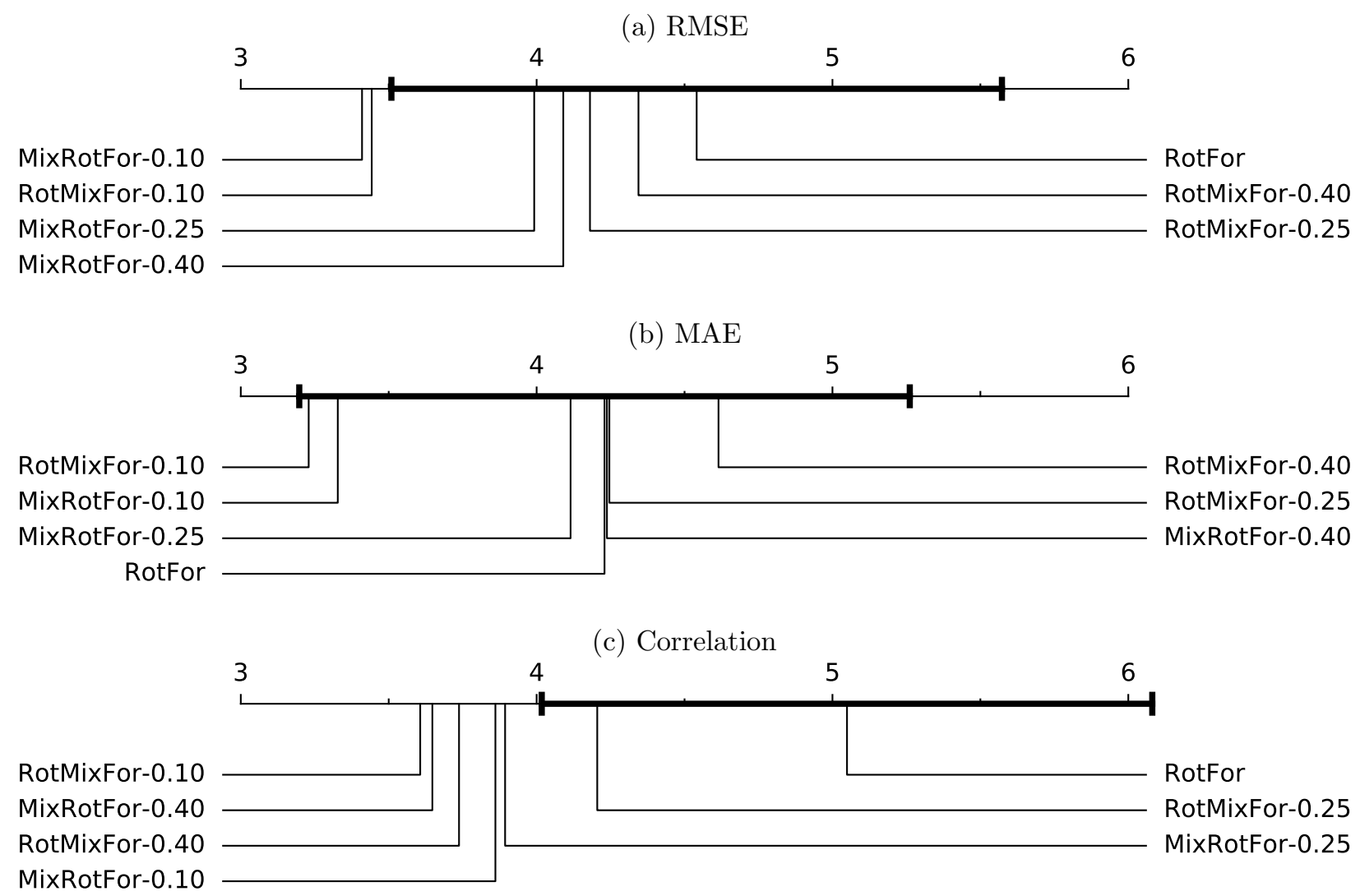

Figure 8: Comparison of Rotation Forest against variants with Mixup, with the Bonferroni-Dunn test. The marked interval spans the critical value and is centered at the mean rank for Rotation Forest. Variants with ranks outside the marked interval are significantly different $(p<0.05)$ from Rotation Forest. 
Table 7: Average ranks.

\begin{tabular}{lr}
\multicolumn{2}{c}{ RMSE } \\
\hline Method & Rank \\
\hline RotMixFor-0.10 & 5.655738 \\
MixRotFor-0.10 & 5.786885 \\
MixRotFor-0.25 & 6.467213 \\
RotMixFor-0.25 & 6.508197 \\
MixRotFor-0.40 & 6.598361 \\
RotMixFor-0.40 & 6.770492 \\
RotFor & 7.016393 \\
MixRandFor-0.40 & 7.893443 \\
MixRandFor-0.25 & 8.196721 \\
MixRandFor-0.10 & 8.221311 \\
RandMixFor-0.10 & 8.418033 \\
RandMixFor-0.25 & 8.893443 \\
RandMixFor-0.40 & 9.040984 \\
RandFor & 9.532787 \\
\hline
\end{tabular}

\begin{tabular}{lr}
\multicolumn{2}{c}{ MAE } \\
\hline Method & Rank \\
\hline RotMixFor-0.10 & 5.581967 \\
MixRotFor-0.10 & 5.827869 \\
RotMixFor-0.25 & 6.762295 \\
MixRotFor-0.25 & 6.811475 \\
MixRotFor-0.40 & 6.950820 \\
RotFor & 7.106557 \\
RotMixFor-0.40 & 7.163934 \\
MixRandFor-0.10 & 7.754098 \\
MixRandFor-0.25 & 7.852459 \\
RandMixFor-0.10 & 7.885246 \\
MixRandFor-0.40 & 8.098361 \\
RandFor & 8.540984 \\
RandMixFor-0.25 & 9.311475 \\
RandMixFor-0.40 & 9.352459 \\
\hline
\end{tabular}

\begin{tabular}{lr}
\multicolumn{2}{c}{ Correlation } \\
\hline Method & Rank \\
\hline RotMixFor-0.10 & 6.016393 \\
RotMixFor-0.40 & 6.114754 \\
MixRotFor-0.40 & 6.188525 \\
MixRotFor-0.10 & 6.418033 \\
MixRotFor-0.25 & 6.549180 \\
RotMixFor-0.25 & 6.795082 \\
MixRandFor-0.40 & 7.778689 \\
RotFor & 7.852459 \\
RandMixFor-0.40 & 7.983607 \\
RandMixFor-0.10 & 8.155738 \\
RandMixFor-0.25 & 8.213115 \\
MixRandFor-0.10 & 8.491803 \\
MixRandFor-0.25 & 8.491803 \\
RandFor & 9.950820 \\
\hline
\end{tabular}

for all the methods together. With regard to RMSE, all the Rotation Forest variants are above all the Random Forest variants. Moreover, the two original methods (without mixup) are the last methods in their respective sets. Likewise, with regard to MAE, the Rotation Forest variants are above all the Random Forest variants, although there are a few variants with mixup below the method without mixup. The methods without mixup for correlation are below all the other methods in their set, although there is some overlap between the two sets, because RandMixFor-0.40 is above RotFor.

Figures 9 and 10 show boxplots for the ranks of the different datasets. Both the Random Forest and the Rotation Forest variants are independently depicted in Figure 9, so the rank values range from 1 to 7 . The Random Forest and the Rotation Forest variants are jointly depicted in Figure 10, so the rank values range from 1 to 14 . These figures support the idea that the use of mixup variants is advisable.

Overall, Rotation Forest shows better performance compared to Random Forest, and mixup offers an advantage for both ensemble methods, which has been empirically demonstrated in our experiment. 
Figure 9: Boxplots for the ranks. The boxplots to the left refer to the Random Forest variants and those to the right refer to the Rotation Forest variants.

(a) RMSE
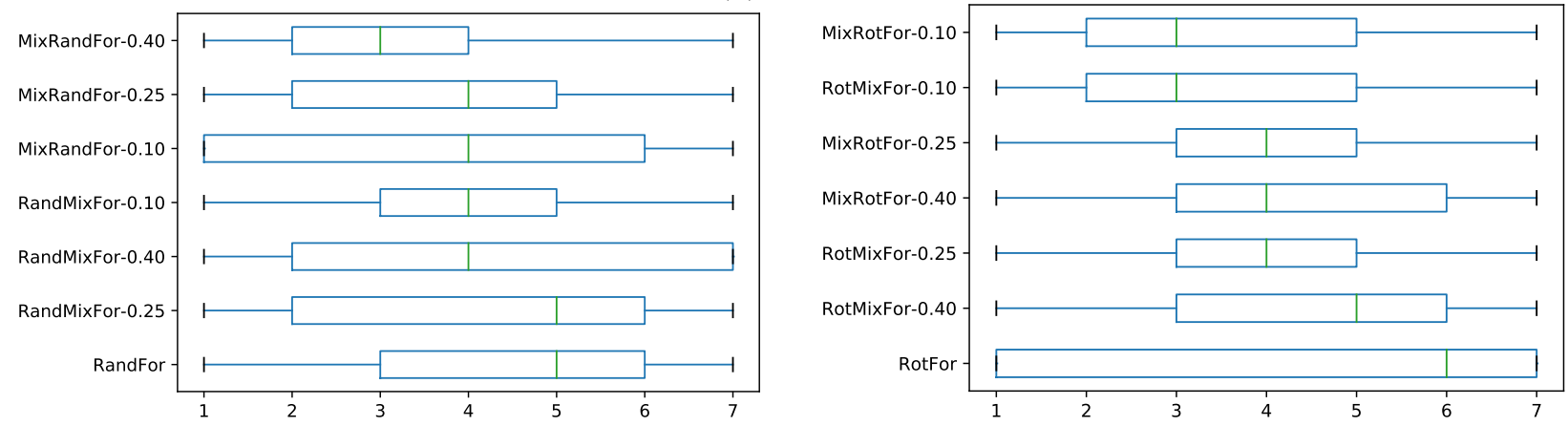

(b) MAE
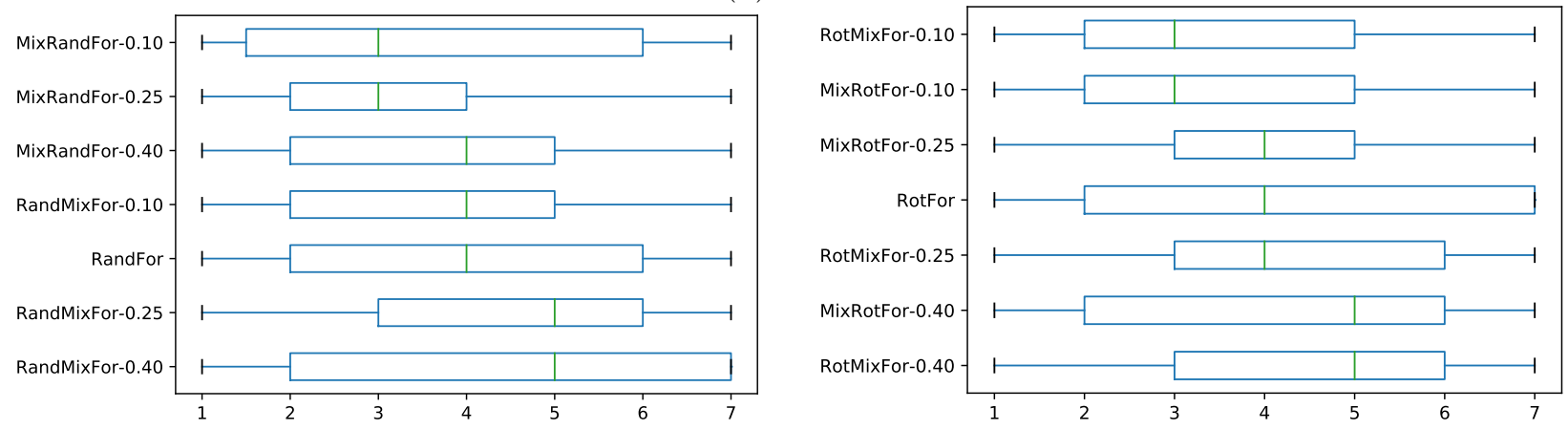

(c) Correlation
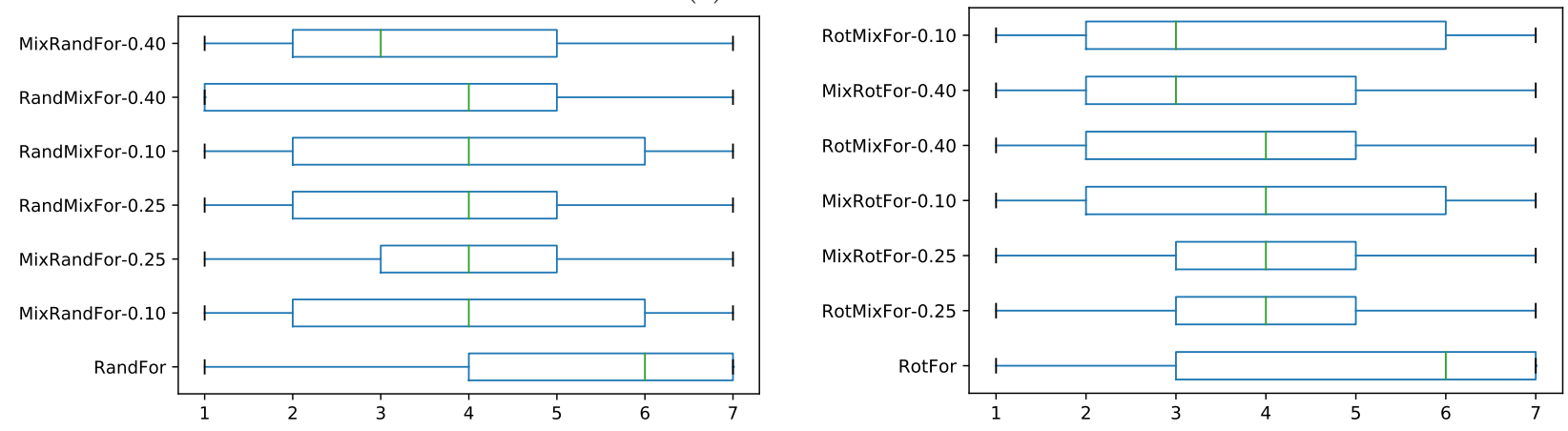
Figure 10: Boxplots for the ranks. The ranks are obtained using both Random and Rotation Forests variants.

(a) RMSE

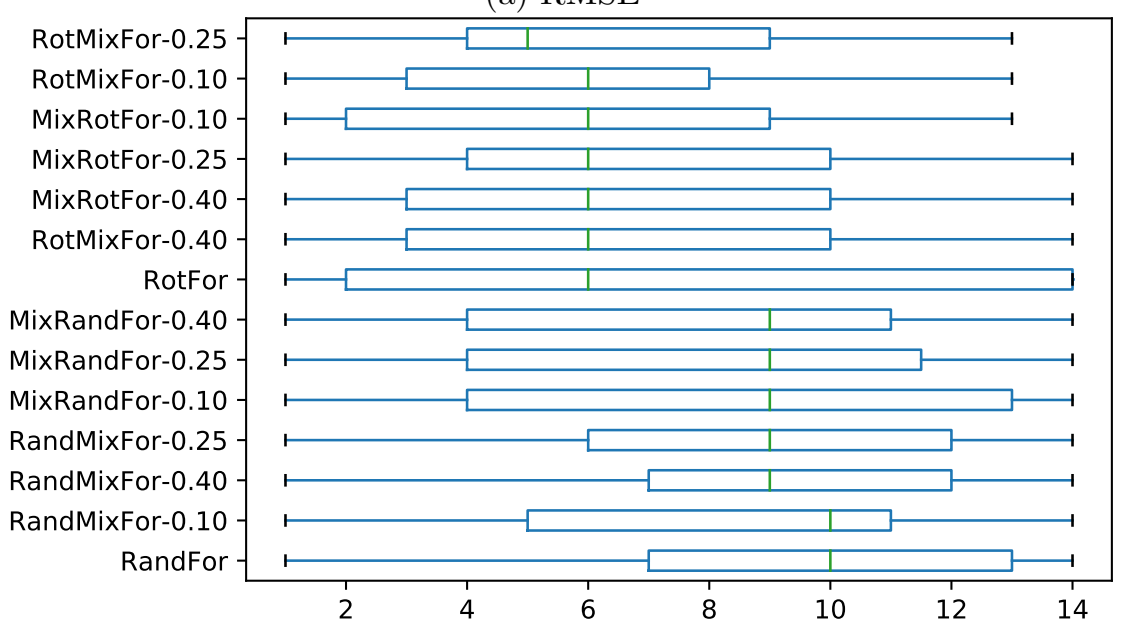

(b) MAE

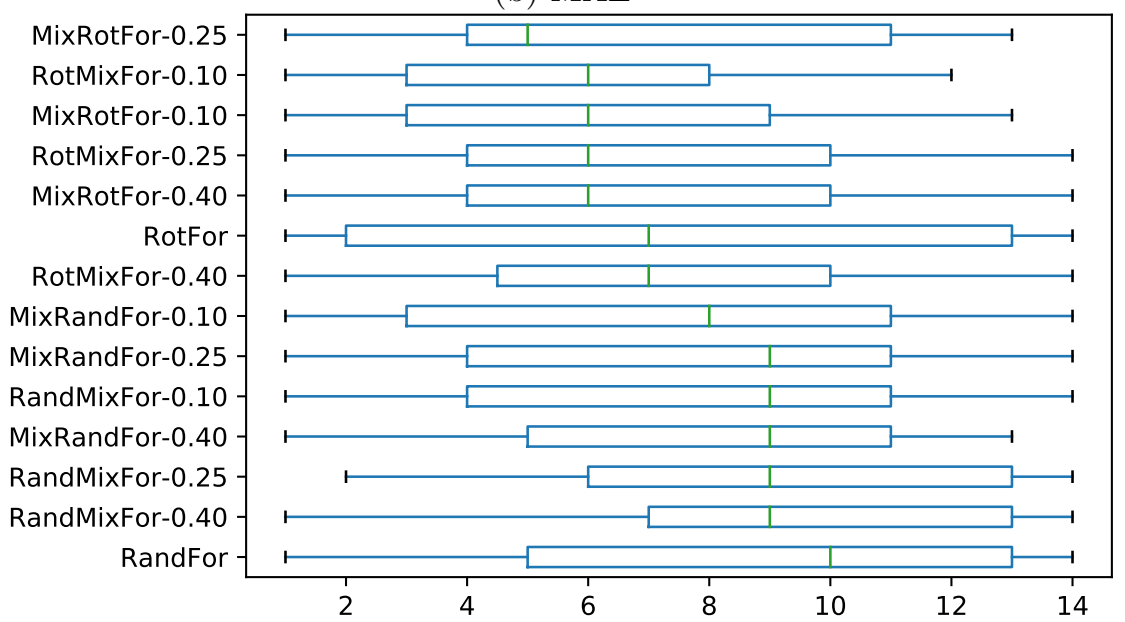

(c) Correlation

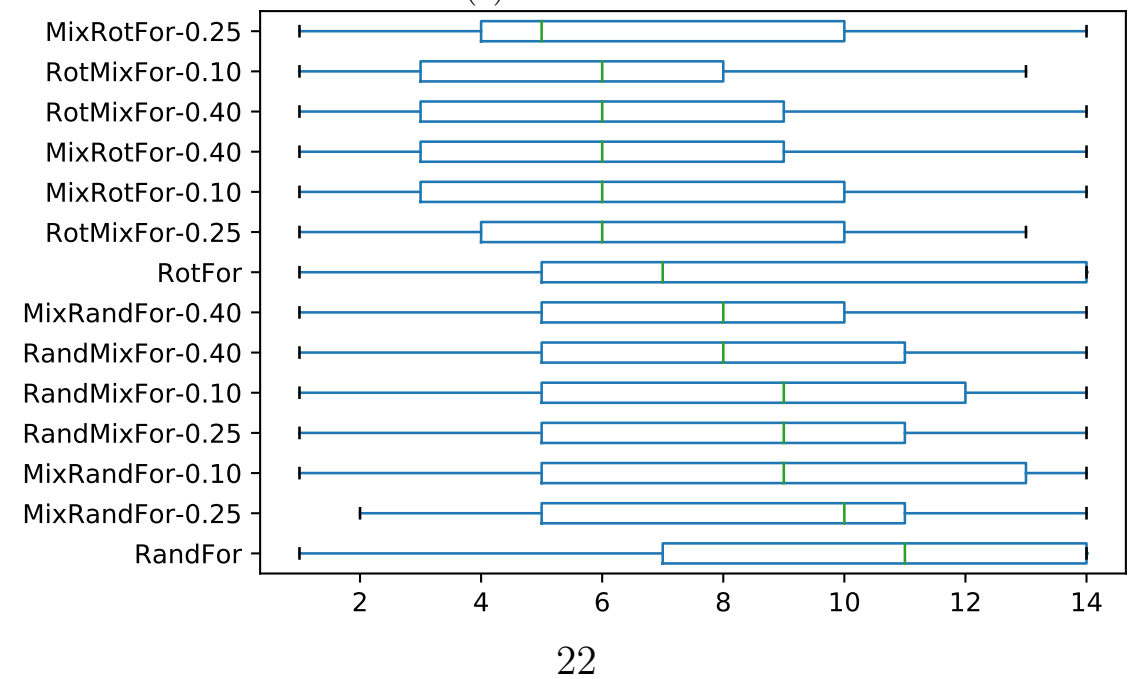


Limitations. The scope of this study is nevertheless limited. The two parameters of the method, the $\alpha$ value for the Beta distribution, and the number of synthetic examples that are generated were not adjusted for each dataset. Only three values of $\alpha$ were considered and the number of synthetic examples was arbitrarily fixed at 50\%. Ensemble size is another parameter that can affect the results and that can interact with the previous parameters. Moreover, the default parameter's values for Random Forest and Rotation Forest were used with no previous adjustment for the study.

The mixup approach has been applied to only two ensemble methods, Random Forest and Rotation Forest, although it could be applied to other methods. For instance, another very successful ensemble method, although not commonly used for regression, is boosting (Solomatine \& Shrestha, 2004). The mixup approach can also be used with ensembles by combining other regression methods rather than classification trees. The usefulness of the mixup approach for regression ensembles with other ensembles and base methods is as yet unproven.

The mixup method was the only method considered for generating artificial instances. Other methods for generating artificial instances might be better suited for a given dataset.

\section{Conclusions and future research}

The mixup strategy has been previously used for regularizing deep neural networks, although this method can also be used for increasing diversity in ensembles. In this paper, we have shown that the performance of regression forest methods can be improved by using the mixup strategy, which introduces artificial instances in the datasets used for training each regression tree. The advantages of the mixup method have been experimentally shown for both Random Forest and Rotation Forest over a broad set of 61 datasets. Our experimental results favored the Rotation Forest and its improved variants.

Some limitations of the study can be approached in future works. The mixup method has one parameter, $\alpha$. We found no clear pattern of influence for the three experimental values $(0.1,0.25$, and 0.4$)$. Adjusting $\alpha$ for each dataset and varying the number of generated artificial instances can both potentially improve the results. 
Mixup forest can be applied to other ensemble methods, such as boosting variants. It can also be used with ensembles formed by other regression models instead of trees.

A future research line is the adaptation of the mixup method for classification datasets. As mentioned earlier, the use of mixup for regression is straightforward, because the output value is continuous. Nevertheless, the application of this method to classification requires a previous decision on the best way of combining different nominal classes. The method could also be useful in problems with several outputs, such as muti-label classification and multi-target regression.

The distribution of the instances can make the mixup strategy counterproductive, because it may add noise in a localized region of the space. With this in mind, further research on the convexity of the space could help clarify the advisability of applying mixup. Moreover, more advanced data augmentation techniques that take into account the manifold of the actual instances would be interesting to explore (Guo et al., 2018; Verma et al., 2018).

Recently, imbalance for regression has been studied (Torgo et al., 2013). The evaluation of whether mixup can be used to work with imbalanced datasets is also a promising area for future research.

\section{Acknowledgments}

This work was supported through project TIN2015-67534-P (MINECO/FEDER, UE) of the Ministerio de Economía y Competitividad of the Spanish Government, project BU085P17 (JCyL/FEDER, UE) of the Junta de Castilla y León (both projects co-financed through European Union FEDER funds), and by the Consejería de Educación of the Junta de Castilla y León and the European Social Fund through a pre-doctoral grant (EDU/1100/2017). The second author is grateful for a Mobility Grant from the Universidad de Burgos. The third author is grateful for a Mobility Grant (CAS19/00100) from the Ministerio de Ciencia, Innovación y Universidades of the Spanish Government. The authors gratefully acknowledge the support of NVIDIA Corporation and its donation of the TITAN Xp GPUs that facilitated this research. 


\section{References}

Bagnall, A., Bostrom, A., Cawley, G., Flynn, M., Large, J., \& Lines, J. (2018). Is rotation forest the best classifier for problems with continuous features? arXiv preprint arXiv:1809.06705,

Beckham, C., Honari, S., Lamb, A., Verma, V., Ghadiri, F., Devon Hjelm, R., \& Pal, C. (2019). Adversarial mixup resynthesizers. arXiv e-prints, .

Breiman, L. (2001). Random forests. Machine learning, 45, 5-32.

Chawla, N., Bowyer, K., Hall, L., \& Kegelmeyer, W. (2002). Smote: Synthetic minority over-sampling technique. Journal of Artificial Intelligence Research, 16, 321-357.

Chawla, N., Lazarevic, A., Hall, L., \& Bowyer, K. (2003). SMOTEBoost: Improving prediction of the minority class in boosting. In 7 th European Conference on Principles and Practice of Knowledge Discovery in Databases( PKDD 2003) (pp. 107-119).

Chen, Z., Chen, W., \& Shi, Y. (2020). Ensemble learning with label proportions for bankruptcy prediction. Expert Systems with Applications, 146,113155. doi:10.1016/j.eswa.2019.113155.

Choi, H., Son, H., \& Kim, C. (2018). Predicting financial distress of contractors in the construction industry using ensemble learning. Expert Systems with Applications, 110, 1 -10. doi:10.1016/j.eswa.2018.05. 026.

Demšar, J. (2006). Statistical comparisons of classifiers over multiple data sets. Journal of Machine learning research, 7, 1-30.

Fernández-Delgado, M., Cernadas, E., Barro, S., \& Amorim, D. (2014). Do we need hundreds of classifiers to solve real world classification problems? Journal of Machine Learning Research, 15, 3133-3181. URL: http://jmlr.org/papers/v15/delgado14a.html.

Frank, E., \& Pfahringer, B. (2006). Improving on bagging with input smearing. In W.-K. Ng, M. Kitsuregawa, J. Li, \& K. Chang (Eds.), Advances in Knowledge Discovery and Data Mining (pp. 97-106). Berlin, Heidelberg: Springer Berlin Heidelberg.

Galar, M., Fernandez, A., Barrenechea, E., Bustince, H., \& Herrera, F. (2012). A review on ensembles for the class imbalance problem: Bagging-, boosting-, and hybrid-based approaches. IEEE Transactions on Systems, Man and Cybernetics Part C: Applications and Reviews, 42, 463-484. doi:10.1109/TSMCC. 2011.2161285 .

García-Pedrajas, N., Pérez-Rodríguez, J., García-Pedrajas, M., Ortiz-Boyer, D., \& Fyfe, C. (2012). Class imbalance methods for translation initiation site recognition in DNA sequences. Knowledge-Based Systems, 25, 22-34. doi:10.1016/j.knosys.2011.05.002.

Geng, G. G., Wang, C. H., Li, Q. D., Xu, L., \& Jin, X. B. (2007). Boosting the performance of web spam detection with ensemble under-sampling classification. In Proceedings - Fourth International Conference on Fuzzy Systems and Knowledge Discovery, FSKD 2007 (pp. 583-587). volume 4. doi:10.1109/FSKD. 
2007.207

Gónzalez, S., García, S., Lázaro, M., Figueiras-Vidal, A. R., \& Herrera, F. (2017). Class switching according to nearest enemy distance for learning from highly imbalanced data-sets. Pattern Recognition, 70, 12-24.

Guo, H., Mao, Y., \& Zhang, R. (2018). Mixup as locally linear out-of-manifold regularization. CoRR, abs/1809.02499. URL: http://arxiv.org/abs/1809.02499.

Haixiang, G., Yijing, L., Shang, J., Mingyun, G., Yuanyue, H., \& Bing, G. (2017). Learning from classimbalanced data: Review of methods and applications. Expert Systems with Applications, 73, 220 - 239. doi:10.1016/j.eswa.2016.12.035.

Hall, M., Frank, E., Holmes, G., Pfahringer, B., Reutemann, P., \& Witten, I. H. (2009). The weka data mining software: an update. SIGKDD Explor. Newsl., 11, 10-18. doi:10.1145/1656274.1656278.

Han, H., Wang, W.-Y., \& Mao, B.-H. (2005). Borderline-smote: A new over-sampling method in imbalanced data sets learning. In D.-S. Huang, X.-P. Zhang, \& G.-B. Huang (Eds.), Advances in Intelligent Computing: International Conference on Intelligent Computing, ICIC 2005, Hefei, China, August 23-26, 2005, Proceedings, Part I (pp. 878-887). Berlin, Heidelberg: Springer Berlin Heidelberg. doi:10.1007/11538059_91.

He, H., Bai, Y., Garcia, E. A., \& Li, S. (2008). ADASYN: Adaptive synthetic sampling approach for imbalanced learning. In 2008 IEEE International Joint Conference on Neural Networks (IEEE World Congress on Computational Intelligence) (pp. 1322-1328). doi:10.1109/I JCNN.2008.4633969.

Inoue, H. (2018). Data augmentation by pairing samples for images classification. CoRR, abs/1801.02929. URL: http://arxiv.org/abs/1801.02929.

Kuncheva, L. I. (2014). Combining pattern classifiers: methods and algorithms. John Wiley \& Sons.

Kuncheva, L. I., \& Whitaker, C. J. (2003). Measures of diversity in classifier ensembles and their relationship with the ensemble accuracy. Machine Learning, 51, 181-207. doi:10.1023/A:1022859003006.

Lindenbaum, O., Stanley, J., Wolf, G., \& Krishnaswamy, S. (2018). Geometry based data generation. In S. Bengio, H. Wallach, H. Larochelle, K. Grauman, N. Cesa-Bianchi, \& R. Garnett (Eds.), Advances in Neural Information Processing Systems 31 (pp. 1400-1411). Curran Associates, Inc. URL: http: //papers.nips.cc/paper/7414-geometry-based-data-generation.pdf.

Marqués, A., García, V., \& Sánchez, J. (2012). Two-level classifier ensembles for credit risk assessment. Expert Systems with Applications, 39, 10916 - 10922. doi:10.1016/j.eswa.2012.03.033.

Martínez-Muñoz, G., \& Suárez, A. (2005). Switching class labels to generate classification ensembles. Pattern Recognition, 38, 1483-1494.

Mayo, M., \& Frank, E. (2017). Improving naive bayes for regression with optimised artificial surrogate data. CoRR, abs/1707.04943. URL: http://arxiv.org/abs/1707.04943.

Melville, P., \& Mooney, R. J. (2003). Constructing diverse classifier ensembles using artificial training 
examples. In IJCAI (pp. 505-510). volume 3.

Melville, P., \& Mooney, R. J. (2005). Creating diversity in ensembles using artificial data. Information Fusion, 6, 99-111.

Menardi, G., \& Torelli, N. (2014). Training and assessing classification rules with imbalanced data. Data Mining and Knowledge Discovery, 28, 92-122. doi:10.1007/s10618-012-0295-5.

Mendes-Moreira, J., Soares, C., Jorge, A. M., \& Sousa, J. F. D. (2012). Ensemble approaches for regression: A survey. ACM computing surveys, 45, 10 .

Panigrahi, S., Kundu, A., Sural, S., \& Majumdar, A. K. (2009). Credit card fraud detection: A fusion approach using Dempster-Shafer theory and Bayesian learning. Information Fusion, 10, 354-363. doi:10. 1016/j.inffus.2008.04.001.

Pardo, C., Diez-Pastor, J. F., García-Osorio, C., \& Rodríguez, J. J. (2013). Rotation forests for regression. Applied Mathematics and Computation, 219, 9914-9924.

Rodríguez, J. J., Kuncheva, L. I., \& Alonso, C. J. (2006). Rotation forest: A new classifier ensemble method. IEEE Transactions on Pattern Analysis and Machine Intelligence, 28, 1619-1630. URL: http: //doi.ieeecomputersociety.org/10.1109/TPAMI.2006.211.

Ross, A., \& Jain, A. (2003). Information fusion in biometrics. Pattern Recognition Letters, 24, 2115 - 2125. doi:10.1016/S0167-8655(03)00079-5.

Sirlantzis, K., Hoque, S., \& Fairhurst, M. (2008). Diversity in multiple classifier ensembles based on binary feature quantisation with application to face recognition. Applied Soft Computing, 8, 437-445. doi:10. $1016 / j$.asoc. 2005.08 .002 .

Soares, S. G., \& Araújo, R. (2015). A dynamic and on-line ensemble regression for changing environments. Expert Systems with Applications, 42, 2935 - 2948. doi:10.1016/j.eswa.2014.11.053.

Solomatine, D. P., \& Shrestha, D. L. (2004). Adaboost.RT: a boosting algorithm for regression problems. In 2004 IEEE International Joint Conference on Neural Networks (IEEE Cat. No.04CH37541) (pp. 11631168 vol.2). volume 2. doi:10.1109/IJCNN. 2004.1380102.

Summers, C., \& Dinneen, M. J. (2019). Improved mixed-example data augmentation. In 2019 IEEE Winter Conference on Applications of Computer Vision (WACV) (pp. 1262-1270). IEEE.

Tay, W.-L., Chui, C.-K., Ong, S.-H., \& Ng, A. C.-M. (2013). Ensemble-based regression analysis of multimodal medical data for osteopenia diagnosis. Expert Systems with Applications, 40, $811-819$. doi:10.1016/j.eswa. 2012.08.031.

Tokozume, Y., Ushiku, Y., \& Harada, T. (2017). Learning from between-class examples for deep sound recognition. arXiv preprint arXiv:1711.10282, .

Tokozume, Y., Ushiku, Y., \& Harada, T. (2018). Between-class learning for image classification. In Proceedings of the IEEE Conference on Computer Vision and Pattern Recognition (pp. 5486-5494). 
Torgo, L., Ribeiro, R. P., Pfahringer, B., \& Branco, P. (2013). Smote for regression. In L. Correia, L. P. Reis, \& J. Cascalho (Eds.), Progress in Artificial Intelligence (pp. 378-389). Berlin, Heidelberg: Springer Berlin Heidelberg.

Verma, V., Lamb, A., Beckham, C., Najafi, A., Mitliagkas, I., Courville, A., Lopez-Paz, D., \& Bengio, Y. (2018). Manifold mixup: Better representations by interpolating hidden states. arXiv preprint arXiv:1806.05236, .

Wang, S., \& Yao, X. (2009). Diversity analysis on imbalanced data sets by using ensemble models. In Computational Intelligence and Data Mining, 2009. CIDM'09. IEEE Symposium on (pp. 324-331). IEEE.

Weng, B., Lu, L., Wang, X., Megahed, F. M., \& Martinez, W. (2018). Predicting short-term stock prices using ensemble methods and online data sources. Expert Systems with Applications, 112, 258 - 273. doi:10.1016/j .eswa.2018.06.016.

Zhang, H., Cissé, M., Dauphin, Y. N., \& Lopez-Paz, D. (2017). mixup: Beyond empirical risk minimization. CoRR, abs/1710.09412. URL: http://arxiv.org/abs/1710.09412v2.

Zhu, T., Lin, Y., \& Liu, Y. (2017). Synthetic minority oversampling technique for multiclass imbalance problems. Pattern Recognition, 72, 327 - 340. URL: http://www.sciencedirect.com/science/article/ pii/S0031320317302947. doi:10.1016/j . patcog. 2017.07.024. 